\title{
A Numerical Study on Particle Tracking and Heat Transfer Enhancement in a Solar Cavity Receiver
}

\author{
C. Ophoff ${ }^{1}$, N. Ozalp ${ }^{2 *}$ D. Moens ${ }^{1}$ \\ ${ }^{1}$ Department of Mechanical Engineering, KU Leuven, 3001 Leuven, Belgium \\ ${ }^{2}$ Department of Mechanical and Civil Engineering, Purdue University Northwest, 46323 Hammond, Indiana, USA
}

\begin{abstract}
The thermochemical dissociation of natural gas into hydrogen and carbon involves the presence of solid phase carbon particles. In addition to the produced carbon, carbon particles can be seeded to the process as a catalyst which act both as a heat transfer enhancing medium and nucleation site for the dissociation reaction. However, presence of discrete phase particles imposes various challenges such as deposition on solar reactor walls and windows, and clogging of the exhaust. Meticulous reactor-receiver design optimization is therefore required and is presented in this study. The Discrete Phase Model (DPM) was implemented in a numerical CFD model to track injected carbon particles in a Lagrangian framework. Validation of the model was done with respect to available experimental data and showed similar deposition phenomena on the reactor window. A parametric design study was performed taking into account flow rates, particle size, particle loading and receiver geometry. Use of a finer particle size distribution demonstrated the ability of the flow to entrain more particles to the exhaust and reduce deposition on the bottom wall of the cavity. Three design iterations on an existing $1 \mathrm{~kW}$ solar cavity receiver were proposed, depicting an increase of at least $6.5 \%$ on the outlet gas temperature and $4 \%$ on the overall average gas temperature within the receiver. Results of this study highlighted the importance of flow configuration, flow parameters and particle size on the deposition and heat transfer inside solar cavity receivers. Outcome of this study can serve as a reference for solar reactor design involving presence of particulate media.
\end{abstract}

Keywords: Solar Cavity Receiver; Particle Tracking; CFD; DPM; Reactor Design 


\section{INTRODUCTION}

With the ever-increasing interest in concentrated solar energy, developments in the field of Concentrated Solar Power (CSP) have brought the estimated share of CSP to the global electricity supply to 7\% by 2030 and $25 \%$ by 2050 [1]. While these commercially adopted solar plants are beneficial from an ecological standpoint as well as from their low operating costs and high efficiency rates, from a transportation and storage standpoint it leads to geographical maldistribution between electricity consumers and the locations where sunshine can effectively be harnessed [2]. This is where solar thermochemical processes can provide a valuable alternative. Comparison of CSP with solar thermochemical processes is visually presented in Figure 1. CSP technologies (Figure 1a) comprise central tower receivers, parabolic trough collectors and parabolic dish concentrators which utilize concentrated solar radiation as the driving force in their Brayton, Rankine and Stirling cycles. Many studies have worked on the optimization of the individual system components of these setups, leading to overall improvement of the solar thermal processes involved. For example, Daabo et al. performed several optimization studies and parametric analyses on the solar cavity receiver geometry and design configuration as the main component of a solar powered Brayton cycle [35]. Another recent study on parabolic trough concentrators numerically investigated the use of a cubicalrectangular cavity receiver rather than conventional tubular receivers with the aim to maximize thermal efficiency [6]. Results of that study provided geometric guidelines with respect to ideal aperture area and cavity depth.

Solar thermochemical processes on the other hand, as depicted in Figure 1b, allow to use concentrated solar radiation as the heat source for highly-endothermical processes yielding solar fuels and commodities as reaction products. These processes would otherwise obtain heat from burning feedstock when the reaction products are obtained from conventional/industrial processes. Advantage of these solar fuels is that they can easily be transported over long distances without degrading. One of these solar thermochemical processes is the thermal dissociation, or direct thermal cracking, of natural gas (i.e. methane). 

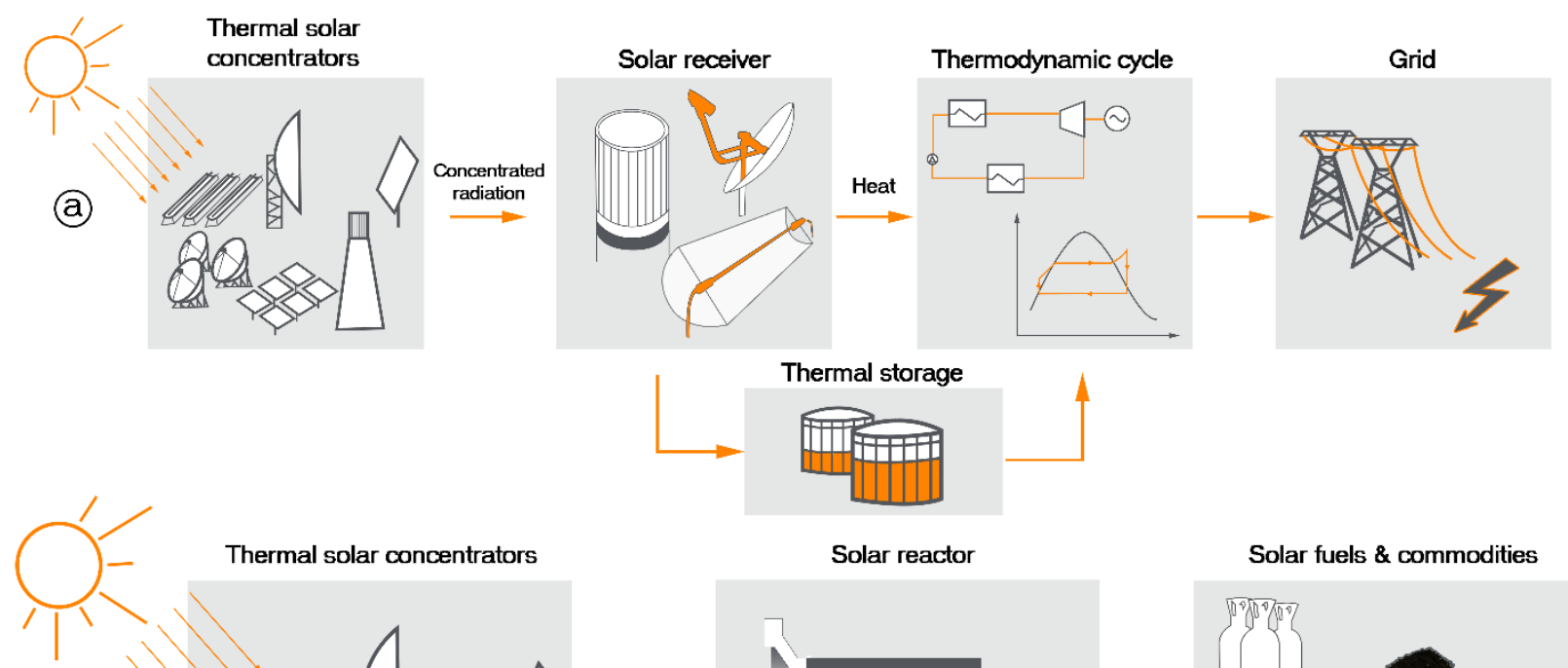

(b)
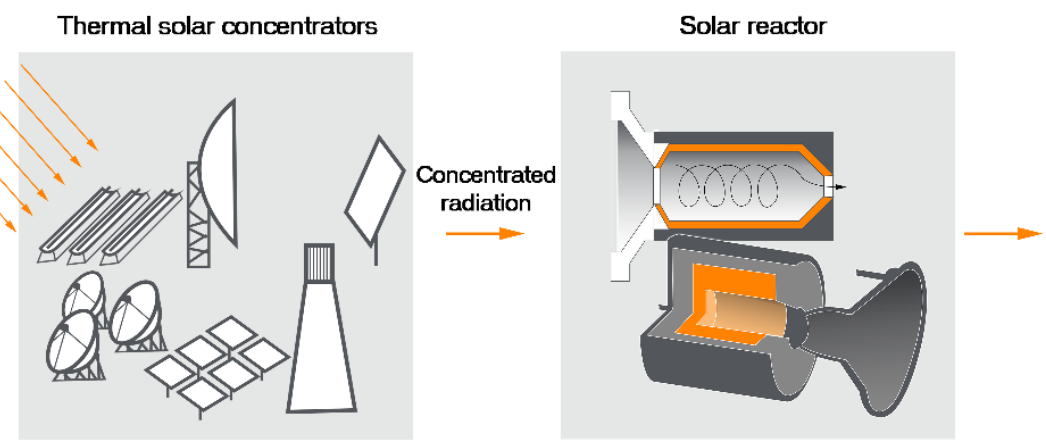

Solar fuels \& commodities

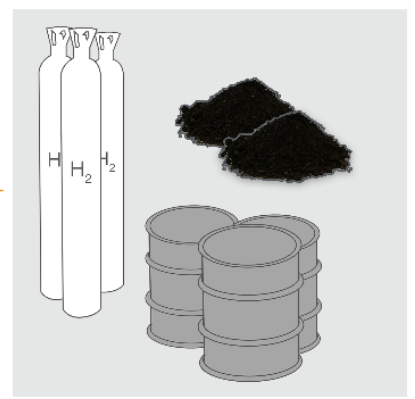

Figure 1 Comparison of CSP with solar thermochemical processes: (a) schematic illustration of a common CSP cycle versus (b) a typical solar thermochemical process.

The solar thermochemical dissociation of methane is characterized by the overall reaction [7]:

$$
\mathrm{CH}_{4} \rightarrow \mathrm{C}+2 \mathrm{H}_{2} \quad\left(\Delta \mathrm{H}^{0}=75.6 \mathrm{~kJ} / \mathrm{mol}\right)
$$

The products of this process are solid Carbon Black (CB) and energy carrier $\mathrm{H}_{2}$. While the latter can be used in fuel cells, $\mathrm{CB}$ has promising industrial applications and above all is obtained free from $\mathrm{CO}_{2}$ that would otherwise be emitted by the conventional furnace process. The activation energy of this reaction varies as it depends on reactor type and, more specifically, its' flow conditions such as residence time, temperature etc. Values of this energy are typically in the range of $200-400 \mathrm{~kJ} / \mathrm{mol}$ [7]. With the use of a particulate catalyst the chemical reaction is upgraded to a heterogeneous reaction and, thus, lowering the activation energy required by the methane decomposition. Although both metal and carbon catalysts can be used, the former has shown to be less effective in terms of $\mathrm{H}_{2}$ yield [8]. The use of carbonaceous catalysts typically results in an activation energy in the range of approximately $150 \mathrm{~kJ} / \mathrm{mol}$ for activated carbons [9] up to approximately $240 \mathrm{~kJ} / \mathrm{mol}$ for carbon black based catalysts [10].

The use of particles and particulate catalysts has widely been adopted in solar thermochemical research. Not only for the thermochemical dissociation of methane, but also for solar water splitting reactions, metaloxide reduction and solar reforming. Brkic et al. modeled and experimentally tested the solar carbothermal 
reduction of aerosolized $\mathrm{ZnO}$ particles with the use of carbon particles [11]. With respect to these particles they observed that enlarging the hot reaction zone in their indirectly heated drop-tube solar reactor would positively affect the reaction as the particle residence time would increase. Residence time of particle catalysts has commonly been a topic of interest in the solar thermochemical field due to its positive impact on chemical conversion. This enhancement of heat transfer and reaction kinetics was for example experimentally shown by one of the earlier studies by Maag et al. where micron size carbon particles were fed to the main $\mathrm{CH}_{4}$ feedstock [12]. Another particle study numerically determined the optimum flow configuration for a solar vortex reactor aimed at maximizing residence time of the particles [13]. Other similar studies in the field evaluated the performance of metallic and carbonaceous catalysts on the solar decomposition of methane [14]. Key outcomes of their research pinpointed the higher reaction rates, enhancement of process efficiency and reduction of other hydrocarbon formation when such particulate catalysts are fed to the gas medium.

Although the solar thermal decomposition of methane is a viable and renewable alternative for the production of hydrogen and carbon black, the process itself encompasses multiple technical challenges preventing the smooth transition to continuous operation and further upscaling of the process. One of these challenges is due to carbon black [15]. Presence of solid carbon particles in the reactor results in deposition on the cavity walls and the window. As deposition increases due to agglomeration and clustering of the particles they can eventually lead to clogging of the reactor exhaust. Secondly, deposition on the quartz window can block incident radiation. Simultaneously, particles attached to the window surface yield elevated temperatures of the window which can further escalate to breakage and cause safety issues of the operation. The above phenomena have extensively been studied by Kogan et al. In their work on solar thermal methane splitting the depositing nature of carbon black is highlighted in terms of the reactor exit port clogging and deposition on the receiver window [16]. Extension of this work showed similar phenomena at room temperature when the reactor was seeded with flow of larger sized carbon particles. Moreover, a tornado flow reactor design was proposed that would drastically diminish deposition on the walls and cavity window [17]. By experimentally visualizing the internal reactor flow dynamics, flow conditions were suggested that would allow to recreate such tornado flow and be effectively adopted in similar reactor-receiver designs [18].

In a study by Rutten and Verschoren the driving mechanisms behind the deposition were listed [19]. They concluded that besides temperature-independent effects such as eddy diffusion, turbulent impactation or gravitational settling, the main driving force is due to the high temperature gradients within the reactor itself. This force, referred to as thermophoretic force, makes the heated carbon particles migrate towards colder regions [20]. More specifically, the cavity and exhaust walls and above all, the reactor window. 
Carbon deposition has also been experimentally observed in different methane cracking reactor types such as the ones found in Ref. [13] and Ref. [16]. Steps have been undertaken towards preventing reactor clogging and window deposition by implementing tornado flow configuration [21] and wall screening [22]. In their numerical study on a solar vortex reactor Chinnici et al. presented two aerodynamic mechanisms in an attempt to address window deposition in solar expanding-vortex receiver-reactors [13]. Outcome of their particle-tracking study yielded ideal geometric and flow configuration parameters with respect to mitigation of particle deposition on the window.

Still, there is room for further improvement of flow and reactor design in order to tackle the technical challenges associated with carbon particles, as elaborated above. While most numerical studies either focused on the flow dynamics, heat transfer or even the chemical reaction involved [23], only limited studies in literature have simultaneously addressed the heat transfer enhancement of particle suspended flows and the resulting deposition of such particles in solar thermochemical reactors [24]. Hence, this study addresses this challenge and aims at improving heat transfer performance inside the reactor cavity while keeping deposition to a minimum. A numerical model is developed that uses the Discrete Phase Model (DPM) to track carbon particles in the flow medium. The results are qualitatively evaluated in terms of deposition and compared to the experimental findings by Kogan et al. [17]. Subsequently, the effect of particle seeding on the heat transfer inside the reactor is investigated and few design iterations of the solar cavity receiver used in this study result in suggested improvements.

\section{CARBON PARTICLE-LADEN FLOW}

\subsection{The heat transfer mechanism}

Similar to falling particle receivers which use particles to increase the solar-to-thermal efficiency in CSP [25], the particle clouds in solar reactors improve the kinetics and heat transfer inside the solar reactor is because of the volumetric gas heating. The particle suspension that is spread throughout the reaction chamber is directly heated by the incident radiation coming from the concentrator. Figure 2 illustrates the difference between an unseeded and a seeded solar reactor. Where an unseeded reactor would mainly transfer heat to the quasi-transparent methane gas through convection from the wall, the suspended particles in a seeded reactor provide an increased heat transfer surface area to the gas. In addition, this heat transfer mechanism is volumetrically spread inside the chamber instead of occurring at the walls only, as would be the case in an unseeded reactor. In conclusion, the carbon particles act as radiant absorbers but also as nucleation sites for the heterogeneous decomposition reaction [26]. 
(a)

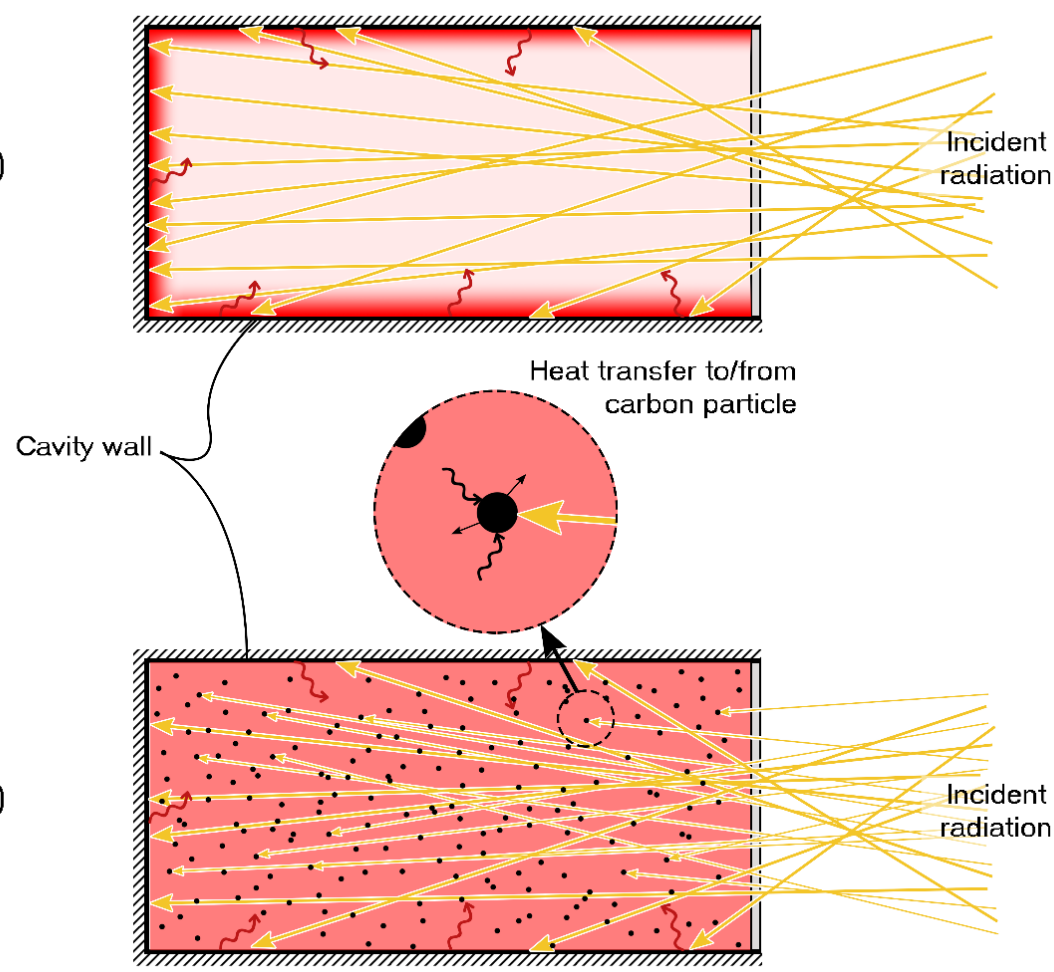

(b)

Figure 2 Illustrative example of (a) an unseeded versus and (b) a seeded solar reactor.

\subsection{The $1 \mathrm{~kW}$ solar cavity receiver}

The parametric design improvement of the present study revolves around a $1 \mathrm{~kW}$ solar cavity receiver that is shown in Figure 3. It consists of a cylindrical cavity with an internal diameter of $12 \mathrm{~cm}$, a 316L stainless steel shell with a $1.5 \mathrm{~cm}$ thickness and wrapped with ceramic fiber insulation $\left(t_{\text {ins }}=3 \mathrm{~cm}\right)$. Three inlets with an inner diameter of $9.25 \mathrm{~mm}$ are tangentially positioned at an angle of 30 degrees with the outer surface normal to the receiver and connected with an inlet manifold. Hence, the main feedstock is equally distributed and develops a vortex flow pattern inside the cylindrical receiver. A set of $11 \mathrm{~K}$-type thermocouples are used to monitor the cavity wall temperature, the cavity gas temperature and the exhaust gas temperature at different locations alongside the receiver. More details on the geometrical specifications, thermocouple configuration and material properties can be found in Ref. [27]. The goal of the present study is to redesign this solar cavity receiver, starting with a validation of the numerical model. 

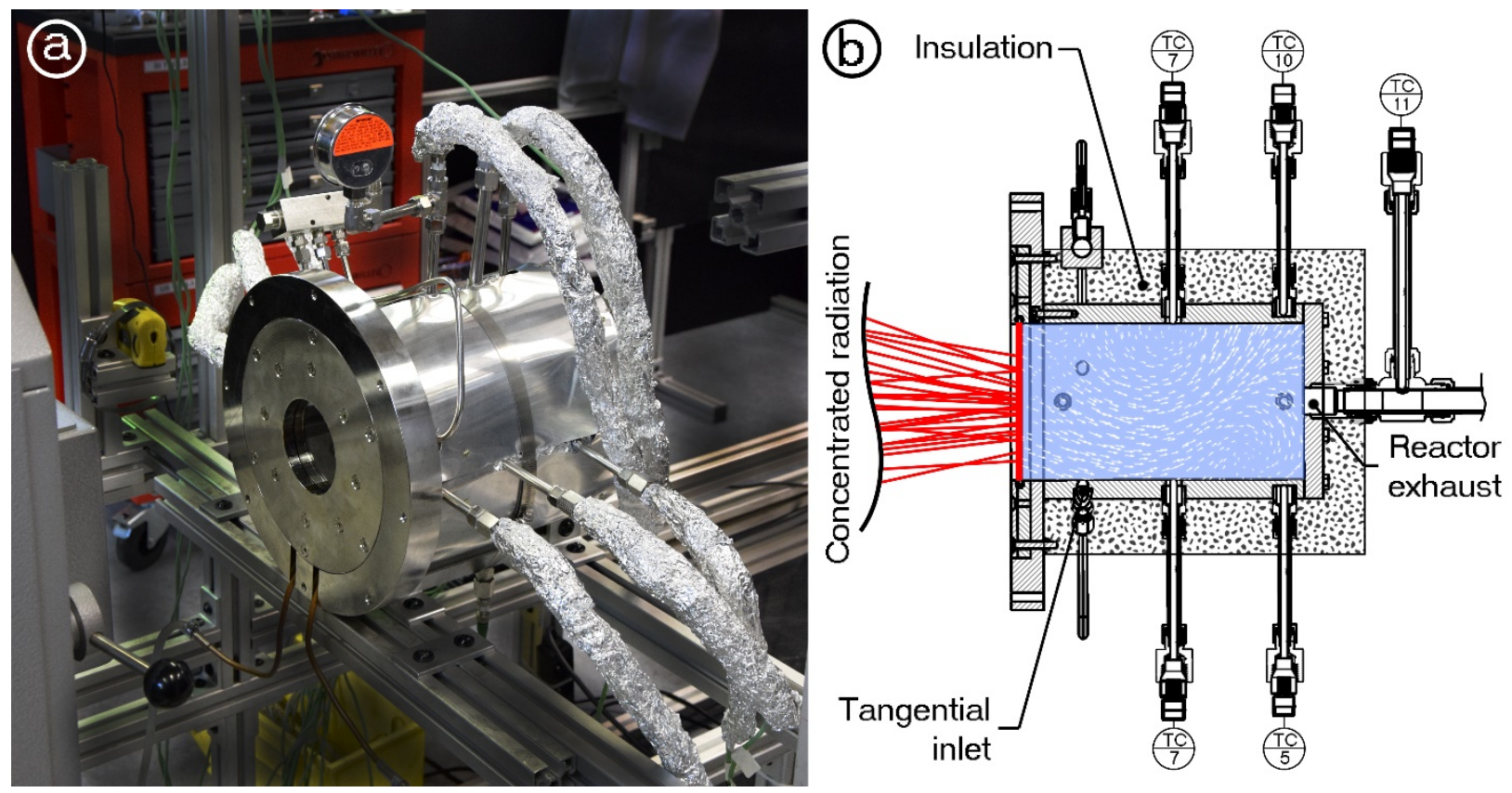

Figure 3 (a) Photo and (b) schematic of the $1 \mathrm{~kW}$ solar cavity receiver configuration.

\subsection{Governing flow equations}

The CFD model is solved using the FLUENT commercial software package [28], which uses the Finite Volume method (FV). Velocity and pressure are coupled through the phase-coupled SIMPLE algorithm which has been used in similar other studies investigating particle-laden flow, such as for example in Ref. [29] and Ref. [24]. The PREssure Staggering Option (PRESTO) scheme is used for the face pressure interpolation, while second order upwind scheme is used for the remaining variables. The convergence criterion of the residual for continuity is set at $10^{-3}$, and $10^{-6}$ for the imbalances of momentum and energy. Achieving the continuity convergence criteria below the $10^{-3}$ residual level was considered sufficient since other quantities of interest showed convergence as well, with the average outlet velocity and average gas temperature being the most important ones. Additionally, the RNG $k-\varepsilon$ turbulence model is used due to the low Reynolds number considered in the case study [17] and the model's ability ability to better capture the effect of swirl flow, as further suggested by similar studies in Ref. [22] and Ref. [24] . The flow does not involve chemically reacting flow but mixing of nitrogen as the main flow and helium as the screening gas is present in the observed case. Therefore, species conservation is enabled in the simulation. The governing equations are given below.

The continuity equation denotes as

$$
\nabla \cdot(\rho \vec{v})=0
$$

The momentum conservation equation is represented by 


$$
\nabla \cdot(\rho \vec{v} \vec{v})=-\nabla p+\nabla \cdot \mu\left[\left(\nabla \vec{v}+\nabla \vec{v}^{T}\right)-\frac{2}{3} \nabla \cdot \vec{v} I\right]
$$

where $p$ is the static pressure, $\mu$ is the molecular viscosity and $I$ is the unit tensor. Gravitational and other external body forces are neglected at this time. The molecular viscosities are equal to $1.99 \times 10^{-5} \mathrm{~kg} / \mathrm{m} . \mathrm{s}$ and $1.663 \times 10^{-5} \mathrm{~kg} / \mathrm{m} . \mathrm{s}$ for Helium and Nitrogen, respectively [30].

Turbulence is modeled via the coupled equations for turbulence kinetic energy $k$ [31]:

$$
\nabla \cdot(\rho k \vec{v})=\nabla \cdot\left(\alpha_{k} \mu_{e f f}\right) \nabla k+G_{k}+G_{b}-\rho \varepsilon
$$

The effective viscosity $\mu_{\text {eff }}$ here is computed using the high-Reynolds-number limit defined as $\mu_{t}=\rho C_{\mu} \frac{k^{2}}{\epsilon}$ with $C_{\mu}=0.0845$. The turbulent kinetic energy dissipation rate $\varepsilon[31]$ :

$$
\nabla \cdot(\rho \varepsilon \vec{v})=\nabla \cdot\left(\alpha_{k} \mu_{e f f}\right) \nabla \varepsilon+C_{1 \varepsilon} \frac{\varepsilon}{k}\left(G_{k}+C_{3 \varepsilon} G_{b}\right)-C_{2 \varepsilon} \rho \frac{\varepsilon^{2}}{k}
$$

The conservation of energy for non-reacting flow with a radiative heat source $Q_{\text {rad }}$ denotes as [31]:

$$
\nabla \cdot(\vec{v}(\rho E+p))=\nabla \cdot\left(k_{e f f} \nabla T+\vec{v} \mu\left[\left(\nabla \vec{v}+\nabla \vec{v}^{T}\right)-\frac{2}{3} \nabla \cdot \vec{v} I\right]\right)+Q_{r a d}
$$

where $k_{\text {eff }}$ is the effective conductivity, $T$ is the static temperature, and the energy $E$ can be represented as $E=h-\frac{p}{\rho}+\frac{v^{2}}{2}$. An overview of the model constants and parameters in the above equations can be found in Ref. [31].

The Discrete Ordinate Radiation Model (DORM) is used to solve the Radiative Transfer Equation (RTE) for a finite number of discrete solid angles. Gray radiation is considered throughout the computational domain, as the wavelength dependence is neglected. Hence, the RTE for an absorbing, emitting and scattering medium at position $\vec{r}$ in the direction $\vec{s}$ is expressed as [31]:

$$
\nabla \cdot(I(\vec{r}, \vec{s}) \vec{s})+\left(a+\sigma_{s}\right) I(\vec{r}, \vec{s})=a n^{2} \frac{\sigma T^{4}}{\pi}+\frac{\sigma_{s}}{4 \pi} \int_{0}^{4 \pi} I(\vec{r}, \vec{s}) \Phi\left(\vec{s} \cdot \vec{s}^{\prime}\right) d \Omega^{\prime}
$$

where $I$ is the radiative intensity, $a$ and $\sigma_{s}$ are the absorption and scattering coefficients both being equal to $1 \times 10^{-9} \mathrm{~m}^{-1}, n$ is the refractive index of the medium and is set to $1, \Phi$ is the phase function which is assigned to be isotropic, $\vec{s}^{\prime}$ is a direction vector, and $\Omega^{\prime}$ is the solid angle. Detailed elaboration of these equations and their constants can be found in Ref. [31].

\subsection{The discrete phase model (DPM)}

The general idea behind the discrete phase model is that the trajectory of individual discrete phase particles ise tracked. Often, bubbles or droplets can be considered instead of particles. In the present study, solid 
carbon particles are used and modeled. The DPM model in FLUENT computes the particle trajectories within a Lagrangian frame of reference. The continuous phase flow, on the other hand, is solved within a Eulerian frame and the exchange of heat, mass and momentum is iteratively computed between both the discrete and continuous phases. Assuming that interaction between the particles in the discrete phase are negligible because of the small particle load, the force balance on the particle relates inertia with the forces acting on the particle [32]. The force balance is written as [31]:

$$
\frac{d u_{p}}{d t}=F_{D}\left(u-u_{p}\right)+\frac{g_{x}\left(\rho_{p}-\rho\right)}{\rho_{p}}+F_{x}
$$

where $F_{D}\left(u-u_{p}\right)$ is the drag force with $F_{D}=\left(18 \mu / \rho_{p} d_{p}^{2}\right)\left(C_{D} R e / 24\right), u$ is the fluid phase velocity which equals to $u=\bar{u}+u^{\prime}$ for turbulent flow, $u_{p}$ is the particle velocity, $\rho$ and $\rho_{p}\left(2000 \mathrm{~kg} / \mathrm{m}^{3}\right)$ are the fluid and particle density, respectively, and $d_{p}$ is the particle diameter which is a function of an assigned distribution. The Reynolds number $R e$ is defined here as [31]:

$$
R e=\frac{\rho d_{p}\left|u_{p}-u\right|}{\mu}
$$

The above-mentioned drag coefficient $C_{D}$ can be obtained in two ways, which are elaborated in [32]. Additional forces on the particle are represented by the term $F_{x}$. Examples of such forces are the thermophoretic force, Brownian force and Saffman's lift force. For the current study $F_{x}$ equals 0 .

In terms of properties and settings for the DPM model the chosen values were mainly based on similar studies found in literature [24], [33] and [34], with a particular focus on the experimental study conducted by Kogan et al. [17]. This study will be used for the validation of the DPM results in the simulations hereafter. Kogan et al. used activated charcoal, i.e. Fluka 05105, in their reactor seeding experiments. However, no information on the particle size and the respective size distribution was reported. Therefore, analogous to the numerical study in Ref. [24], a Rosin-Rammler (R-R) distribution fitted to a similar carbon powder type (Fluka 03866) in Ref. [34] is used in the numerical model. Rosin-Rammler is the mathematical formulation of a Weibull distribution, which is commonly used in particle size analyses. Its name originates from Rosin and Rammler who were the first to apply this Weibull distribution [35]. For the current model, the R-R distribution fits the particle size distribution with a mean particle diameter $d_{p, m}$ of $20 \mu \mathrm{m}$ and a spread of 1.2. The limits are set to a minimum particle diameter $d_{p}$ of $1 \mu \mathrm{m}$ and a maximum diameter $d_{p}$ of $100 \mu \mathrm{m}$. A density $\rho_{d}$ of $2000 \mathrm{~kg} / \mathrm{m}^{3}$ is assigned to the carbon particle material in FLUENT. The particles are modeled as inert discrete phase elements, subject to heating or cooling.

Also in this fluid dynamics model the DORM is used to solve the RTE. To include the particulate effect in the DORM, the radiative transfer equation in Eq. 7 is modified into [31]: 


$$
\nabla \cdot(I(\vec{r}, \vec{s}) \vec{s})+\left(a+a_{p}+\sigma_{p}\right) I(\vec{r}, \vec{s})=a n^{2} \frac{\sigma T^{4}}{\pi}+E_{p}+\frac{\sigma_{p}}{4 \pi} \int_{0}^{4 \pi} I\left(\vec{r}, \overrightarrow{s^{\prime}}\right) \Phi\left(\vec{s} \cdot \overrightarrow{s^{\prime}}\right) d \Omega^{\prime}
$$

where $E_{p}$ is the equivalent emission of the particle cloud and $a_{p}$ is the equivalent absorption coefficient of the particulate flow and can be evaluated using [31]:

$$
a_{p}=\lim _{V \rightarrow 0} \sum_{n=1}^{N} \epsilon_{p n} \frac{A_{p n}}{V}
$$

with $\epsilon_{p n}$ and $A_{p n}$ being the emissivity and projected area of particle $n$, respectively. The emissivity is set to 0.9 while the projected area $i s A_{p n}=\pi d_{p n}{ }^{2} / 4$. In the present study an absorption coefficient $a_{p}$ of $0.5 \mathrm{~m}^{-1}$ is considered [36]. The equivalent scattering coefficient $\sigma_{p}$ in Eq. 10 is defined by [31]:

$$
\sigma_{p}=\lim _{V \rightarrow 0} \sum_{n=1}^{N}\left(1-f_{p n}\right)\left(1-\epsilon_{p n}\right) \frac{A_{p n}}{V}
$$

with $f_{p n}$ being the scattering factor associated with the $\mathrm{n}^{\text {th }}$ particle and is set to to $0.5 \mathrm{~m}^{-1}$. Given that the scattering effect in the particle-suspended flow is dominated by the particles, all other sources of scattering (e.g. $\sigma_{s}$ in Eq. 7) are neglected.

\section{RESULTS \& DISCUSSION}

\subsection{DPM results validation}

To validate the numerical results obtained with the DPM model, the experimental campaigns conducted by Kogan et al. [17] are considered in this study. The fluid domain is defined based on the specifications found in Ref. [17] and Ref. [19]. The reactor used in those studies consists, as illustrated in Figure 4, of a vertically oriented cavity with a quartz window at the top. The main geometry can be described as a combination of a cylinder with $L_{c y l}=140 \mathrm{~mm}$ and a conical frustum bottom part with $L_{c o n}=70 \mathrm{~mm}$ and a diameter-todiameter ratio of 20. Diameter $d_{c y l}$ of the cylindrical body is $140 \mathrm{~mm}$. In terms of flow configuration the reactor has a radial inlet at the very top to protect the window from possible deposition and, hence, referred to as the window screening flow $F_{2}$. The main inlet $F_{l}$ consists of eight tangential inlet ports to induce a vortex like flow inside the cavity. Two additional radial inlets $F_{31}$ and $F_{32}$ are used to protect the wall from possible carbon deposition through generation of a wall screening flow. And finally, seeding of the reactor with carbon particle flow occurs at the respective seeding ports $F_{s 1}, F_{s 2}$ or $F_{s 3}$. 


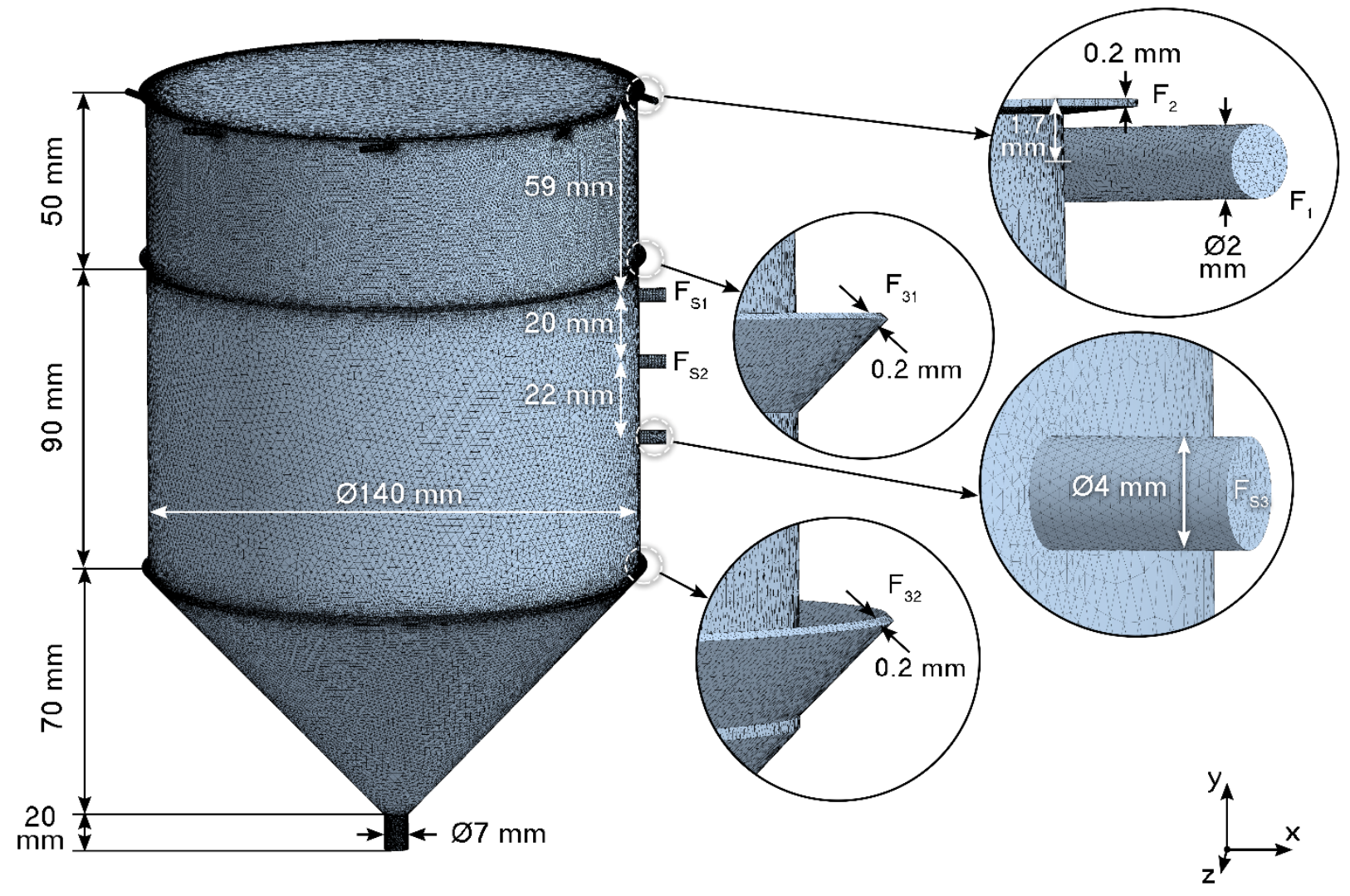

Figure 4 Unstructured mesh and geometric specification of the seeded solar reactor from [17] (2,121,055 cells).

Table 1 Results of the grid independence study for the seeded solar reactor from [17].

\begin{tabular}{lllll}
\hline Grid size & Max. skewness & $\mathbf{V}_{\text {avg,out }}$ & $\mathbf{y}+_{\text {walls,avg }}$ & $\mathbf{y}+_{\text {wall,max }}$ \\
{$[$ [\# cells] } & {$[-]$} & {$[\mathrm{m} / \mathrm{s}]$} & {$[-]$} & {$[-]$} \\
\hline 776,913 & 0.8792 & 5.8393 & 3.22 & 9.28 \\
$1,288,079$ & 0.9140 & 6.1468 & 1.98 & 6.54 \\
$2,121,055$ & 0.8959 & 5.8468 & 1.46 & 4.95 \\
$3,081,683$ & 0.9251 & 5.9848 & 1.10 & 4.20 \\
\hline
\end{tabular}

\subsubsection{Grid independence study}

SolidWorks was used for drawing the three-dimensional fluid domain geometry which was imported in the ANSYS workbench thereafter. An unstructured mesh was then generated with the ANSYS meshing tool for the studied reactor geometry as is depicted in Figure 4. Prior to the full computation of the DPM and energy enabled flow, a flow-only study is conducted to choose the appropriate size of the computational 
grid. Due to the small dimensions of the inlet ports with respect to the overall diameter and length of the cavity, the grid is iteratively adapted to obtain reasonable $y+$ values in order to accurately capture turbulence phenomena at the cavity wall. Results of the grid independence study are summarized in Table 1. Overall, the meshes required local refinements with acceptable growth ratios at the inlet regions to obtain maximum skewnesses within reasonable bounds. Both the average and maximum $y+$ values at the cavity walls were monitored. The grid size of 2,121,055 cells is chosen given that more refinement of the grid would yield further fluctuations of the average exit velocity. In terms of $y+$ the maximum value does not go beyond 4.95 which indicates that the mesh resolution is sufficient for capturing the laminar sub-layer.

\subsubsection{Test parameters overview}

In the study by Kogan et al. different cases were experimentally tested where different flow rates and positions of the seeding ports were compared [17]. A total of 13 experimental seeding simulation tests were conducted. For the sake of brevity, four of these are numerically computed here and the results are mainly qualitatively compared with the experiments. Table 2 shows the parameters used in the 4 cases. As aforementioned, three different seeding port locations are available in the reactor geometry. Therefore, to observe the effect of the seeding inlet on possible carbon deposition, three of the selected tests have a different seeding port. One additional case is chosen to observe the effect of the seeding flow rate, which is approximately a factor 4 larger (test \#1) than the other three cases. The particle loading also changed proportionally with the flow rate. For all the tests conducted by Kogan et al. nitrogen is used for the flows fed at $F_{1}, F_{s 1}, F_{s 2}, F_{s 3}, F_{31}$ and $F_{32}$. Only for the window screening flow $\left(F_{2}\right)$ helium is used instead.

Table 2 Flow parameters of the seeding simulation tests

\begin{tabular}{llllllll}
\hline Test no. & $\mathbf{F}_{\mathbf{1}}$ & $\mathbf{F}_{\mathbf{2}}$ & $\mathbf{F}_{\mathbf{s} 1}$ & $\mathbf{F}_{\mathbf{s} 2}$ & $\mathbf{F}_{\mathbf{s} 3}$ & $\mathbf{F}_{\mathbf{3 1}}$ & $\mathbf{F}_{\mathbf{3 2}}$ \\
& $l / m i n$ & $l / m i n$ & $l / m i n$ & $l / m i n$ & $l / m i n$ & $l / m i n$ & $l / m i n$ \\
\hline$\# 1$ & 9.51 & 1.74 & 0 & 0 & 2.12 & 0 & 0 \\
$\# 4$ & 9.40 & 0.77 & 0 & 0 & 0.54 & 1 & 0 \\
$\# 10$ & 9.62 & 0.80 & 0 & 0.59 & 0 & 1 & 0.9 \\
$\# 12$ & 9.46 & 0.76 & 0.59 & 0 & 0 & 1 & 0.9 \\
\hline
\end{tabular}

\subsubsection{Results and validation}

The numerical results for the respective cases were examined in the ANSYS CFD-Post module [28]. For the volume flow rates given in Table 2 average velocities throughout the fluid domain were obtained in the 
range of 0.25 to $0.27 \mathrm{~m} / \mathrm{s}$. The resulting Reynolds number of the flow is found to be close to 3000 , indicating the low turbulence nature of the flow. Hence, this supports the choice of using the RNG $k-\varepsilon$ turbulence model in the present study. Although species conservation is enabled in the numerical model to distinguish the helium and nitrogen flow, no special attention is focused on the concentration distribution of both flows at steady state. The results only showed that the mass fraction of helium in the fluid domain is mainly concentrated at the inlet of the radial window screening flow but did not spread towards the center of the window region.

Figure 5 shows the resulting particle tracks for all four cases from Table 2. The particle trajectories are colored per particle diameter within the $1-100 \mu \mathrm{m}$ range assigned to the Rosin-Rammler distribution at the seeding port. The blue-colored tracks mainly represent the smaller particles of the carbon feed while the visible green and yellow tracks represent the larger and heavier particles. The flow is able to carry the smaller (i.e. lighter) particles to the top part of the cavity. Kogan et al. described this phenomenon as a toroidal flow perturbation [17]. A friction occurs between the gas stream in the tornado funnel and the gas surrounding it which causes a weak flow perturbation. The streamlines of this perturbed quiescent fluid region, which are helically wrapped around toroidal surfaces, are visually depicted in Figure 6b. As they reach the reactor bottom this flow phenomenon leads to the solid particles being elevated and carried towards the top. Hence, they reach the cavity window and are carried away in the tornado funnel. 


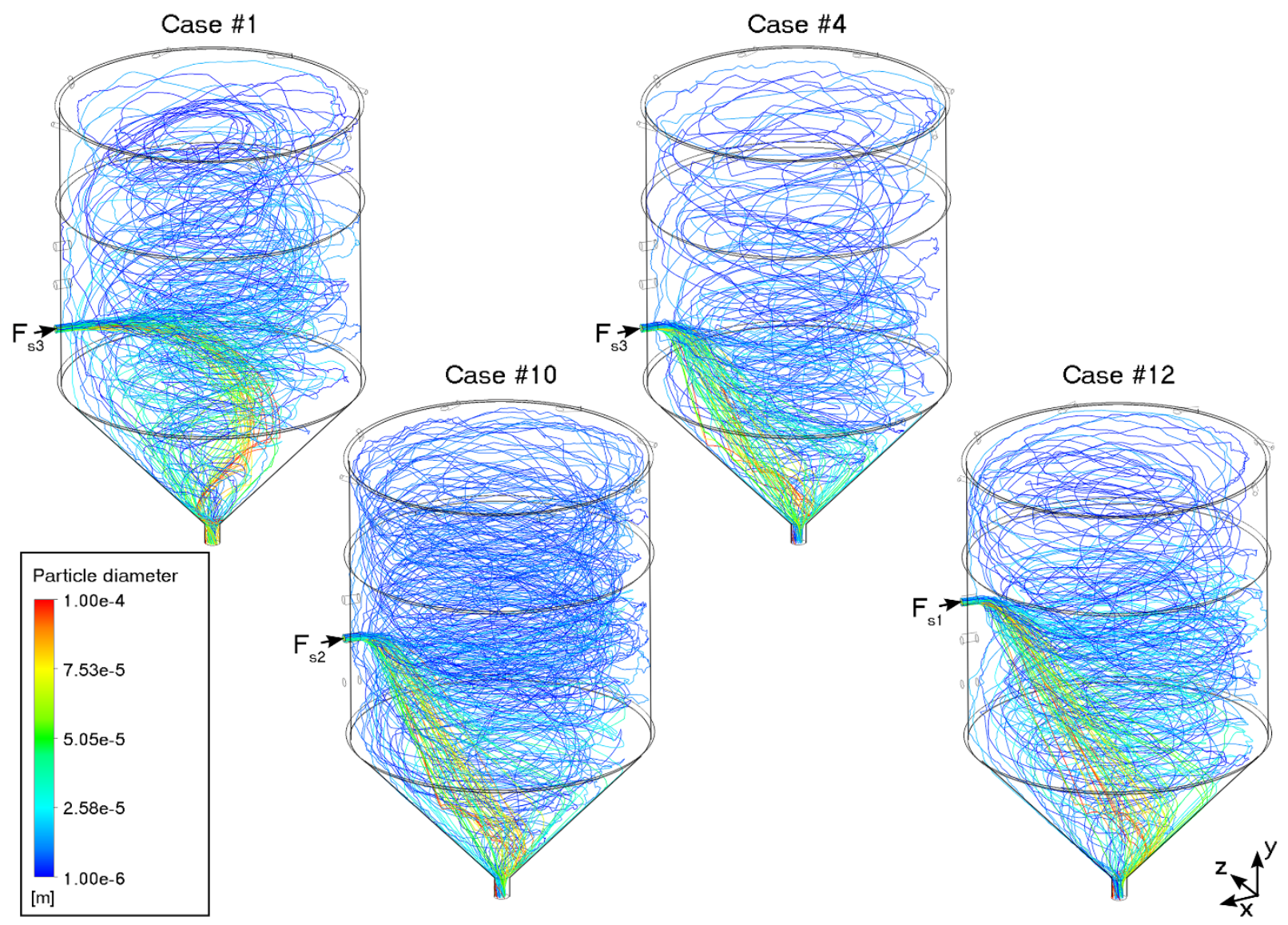

Figure 5 Particle trajectories for all four cases presented in Table 2.

The numerical results in Figure 6a are in agreement with the above described torroidal phenomenon that is illustrated in Figure $6 \mathrm{~b}$ and c. This was the case regardless of the slightly different geometry of the bottom part, i.e. conical frustum versus flat bottom plate. Especially for cases \#4, \#10 and \#12 the flow pattern agrees well. Case \#1 shows a disturbance of the axi-symmetrical flow pattern due to the higher seeding flow rate at $F_{s 3}$. The effect of this higher flow rate, which is a factor four times higher than the other cases, is also observed in the particle trajectories depicted in Figure 5. 
Case \#1

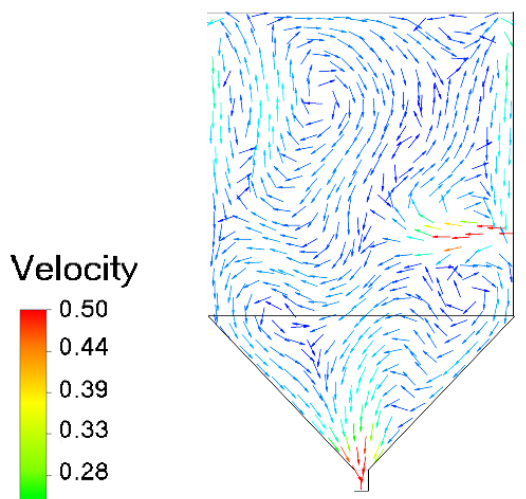

$-0.22$

$-0.17$

0.11

0.06

0.00

$[\mathrm{m} / \mathrm{s}]$

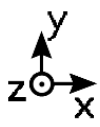

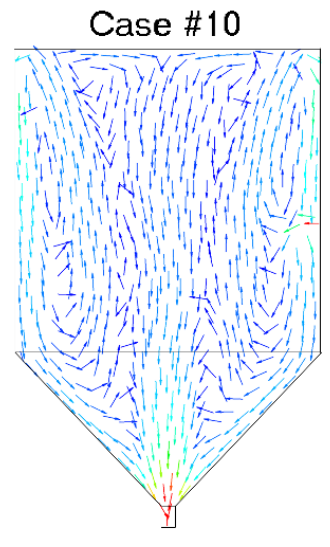

\section{Case \#4}

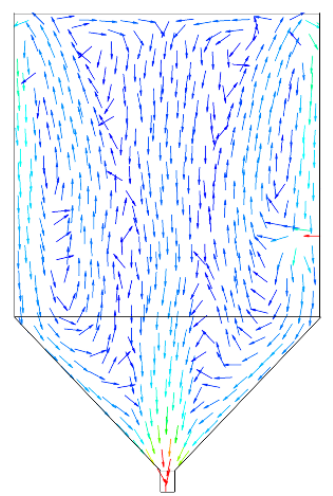

Case \#12

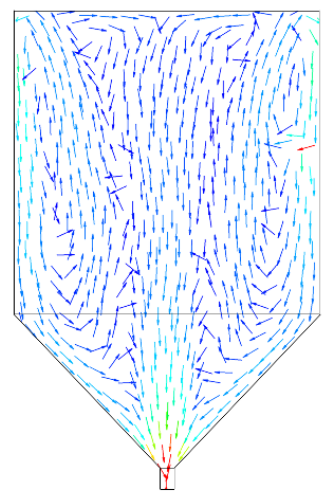

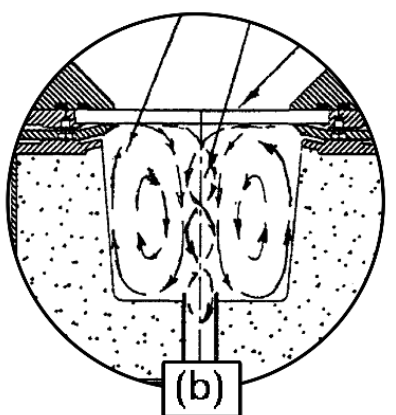

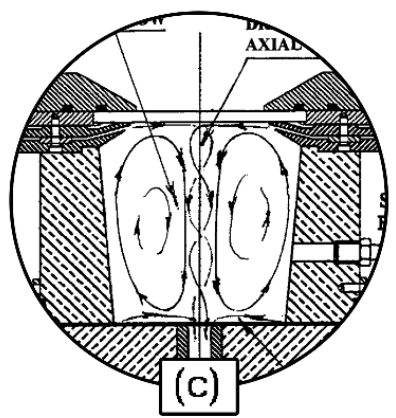

(a)

Figure 6 Comparison of the velocity vector field for all four cases: (a) the numerical results versus (b-c) the presumed schematic representation of the torroidal flow perturbation from [17]. (Reprinted from "Production of hydrogen and carbon by solar thermal methane splitting. II. Room temperature simulation tests of seeded solar reactor", Vol. 29, Kogan A., Kogan M. and Barak S., pp. 1227-1236, Copyright 2004, with permission from Elsevier)

The heavier particles are able to reach the core of the vortex funnel in case \#1. For cases \#4, \#10 and \#12, on the other hand, these particles are driven by gravity as they are pulled down toward the exit port. In order to predict particle concentration throughout the fluid domain with a specific interest of determining deposition on the cavity walls and window, the particle mass concentration is used. Figure 7 shows the DPM particle mass concentration contours on the cavity wall. The contours are viewed from the side of the carbon feeding ports, as indicated in Figure 7 (Case \#1). Discussion of these results can be summarized as follows:

- As can be expected, the conical frustum causes accumulation of the discrete phase particles due to gravitational settling.

- With respect to cases \#4, \#10 and \#12, again, case \#1 shows significantly higher concentration values due to its' increased flow rate and particle loading.

- The presence of a wall screening flow at the conical frustum $\left(F_{32}\right)$ in cases \#10 and \#12 has very little impact on the particle concentration. However, with respect to case \#4 the screening flow in the other 
two cases is able to slightly reduce concentration just beneath the radial inlet. Higher flow rates should be introduced in order to affect the possible deposition lower in the frustum.

- One other observation in the numerical results is the uneven concentration distribution in the bottom part. Due to the counter-clockwise vortex structure, particles are unevenly spread around the frustum part of the reactor. As was previously seen in Figure 5, the larger/heavier particles tend to fall directly into the receiver's bottom part. Presence of the vortex flow entrains the particles a bit, but the highest concentration is observed close to the radial position of the seeding port. This uneven concentration distribution is in agreement with the powder sedimentation that is observed in the experimental campaigns by Kogan et al. [17].
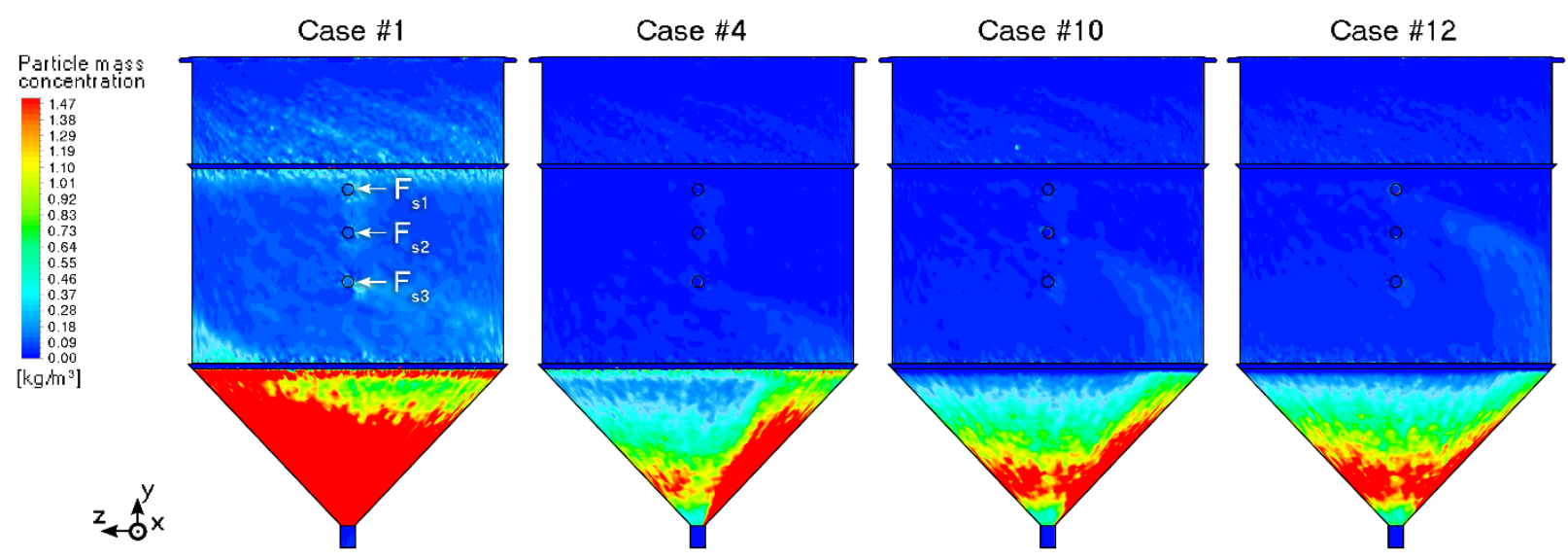

Figure 7 Comparison of the particle mass concentration at the cavity walls for all four cases as a representative quantification of the particle deposition. 
(a)

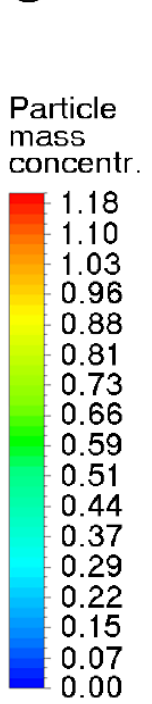

$\left[\mathrm{kg} / \mathrm{m}^{3}\right]$

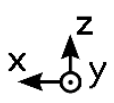

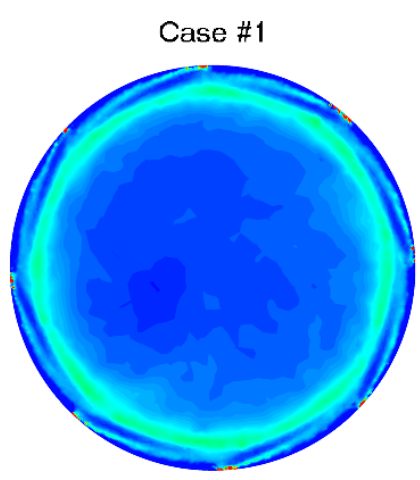

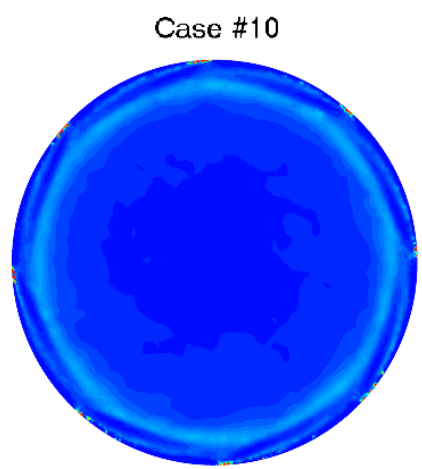

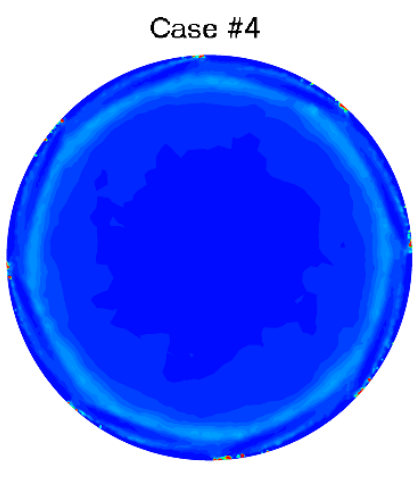

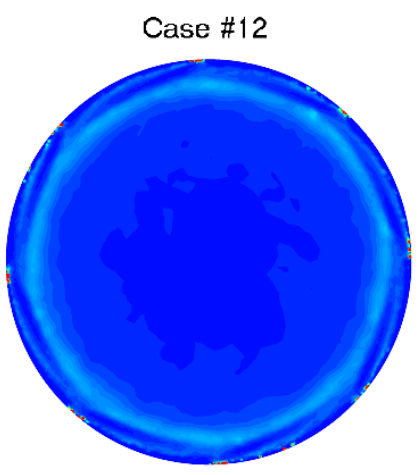

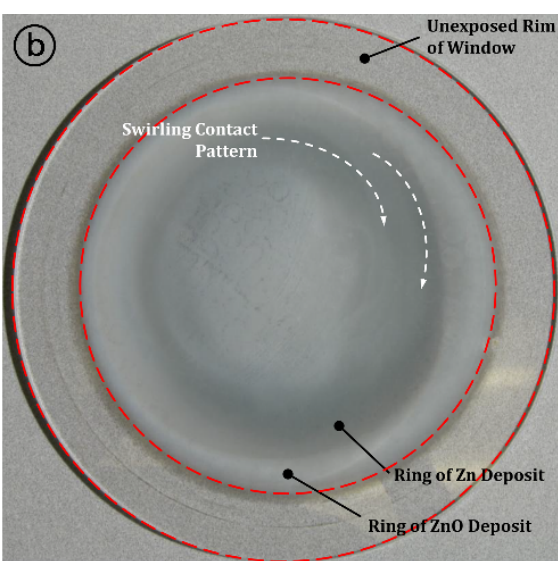

(C)

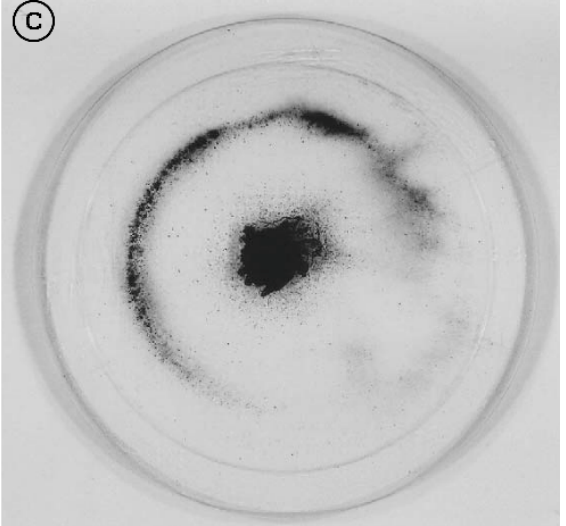

Figure 8 (a) Comparison of the particle mass concentration at the quartz window for all four cases as a representative quantification of the particle deposition; (b) comparable window deposition of $\mathrm{Zn}$ and $\mathrm{ZnO}$ [37]; and (c) experimental results from Kogan et al. [17]. (Reprinted from "Production of hydrogen and carbon by solar thermal methane splitting. II. Room temperature simulation tests of seeded solar reactor", Vol. 29, Kogan A., Kogan M. and Barak S., pp. 1227-1236, Copyright 2004, with permission from Elsevier)

The main goal of the experimental seeding study conducted by Kogan et al. is to provide window screening through the use of the aforementioned tornado flow configuration. This way, the window would be protected from carbon particle contact which might escalate into breakage of the window. Their qualitative results by visual inspection of the window state described the presence or absence of carbon powder. Moreover, they used grease to wet the surface and retain particle that would make contact with the window. Similar to the particle mass concentration results presented above, the window is also studied in the fluid dynamics model solution. Results are shown in Figure 8. In agreement with the particle concentration results and trajectories in Figure 5 and Figure 7 concentration at the window is considerably higher for case $\# 1$. Also here, the effect of the vortex driven by the tangential inlets is visible as seen in the concentrically shaped circle (Figure 8a and c). Not only is this shape observed in the experimental study by Kogan et al., but also a similar pattern as found in the doctoral study by Koepf[37]. Koepf studied the solar thermal decomposition of $\mathrm{ZnO}$ and adopted the vortex generation system from Kogan et al. in his reactor design. In one of his experimental campaigns performance of the vortex generation is ineffective, causing deposition 
of $\mathrm{Zn}$ and $\mathrm{ZnO}$ on the window and, thus, terminating the operation of the reactor. Closer observation at the fouled window (Figure 8b) showed a similar concentric deposition pattern as is observed in the present simulations. Nevertheless, the central spot of $\mathrm{CB}$ deposition in Figure $8 \mathrm{c}$ did not repeat itself in the numerical simulations nor in the experimental campaigns by Koepf. Plausible explanation would be the reactor geometry. The conical frustum in both the numerical model and the reactor studied by Koepf would result in a weaker upwelling stream which would not be able to deposit the particles in the central region of the window.

\subsection{Carbon particle seeding of the $1 \mathrm{~kW}$ receiver}

Overall, the results and validation in the previous section sufficiently agree in order to use the DPM model and apply it to the $1 \mathrm{~kW}$ solar cavity receiver presented in section 2.2. The flow configuration and dimensions were previously given. Since only the main flow is present, i.e. main feedstock through 3 inlet ports at the front of the receiver, the carbon particle feed is considered suspended in this stream for this numerical study. This way good mixing of the flow and particles is guaranteed. For the DPM settings the same Rosin-Rammler distribution is taken for this numerical computation and the same inlet conditions were assumed for all three inlet ports. No additional window or wall screening flow is introduced.

\subsubsection{Particle trajectories and concentration}

The results of the DPM-enabled computation are shown in Figure 9. Opposed to the vertically oriented receiver from the Weizman Institute of Science (WIS) presented above, the $1 \mathrm{~kW}$ solar cavity receiver is designed to be operated in horizontal orientation. This significantly affects the followed paths of the discrete particles. As before, gravity has a major impact on the heavier particles and causes them to fall down close the axial position of the inlets, as seen in Figure 9a. While the smaller CB particles are light enough to be entrained downstream through the outlet, a big portion of the larger/heavier particles remain at the bottom of the cavity. Hence, particles accumulation occurs in the front part of the receiver as shown in the bottomview perspective in Figure 9b. It is striking, however, to ascertain that the window remains relatively clean with respect to the cavity walls given the fact that no window screening flow is involved in the present flow configuration. 


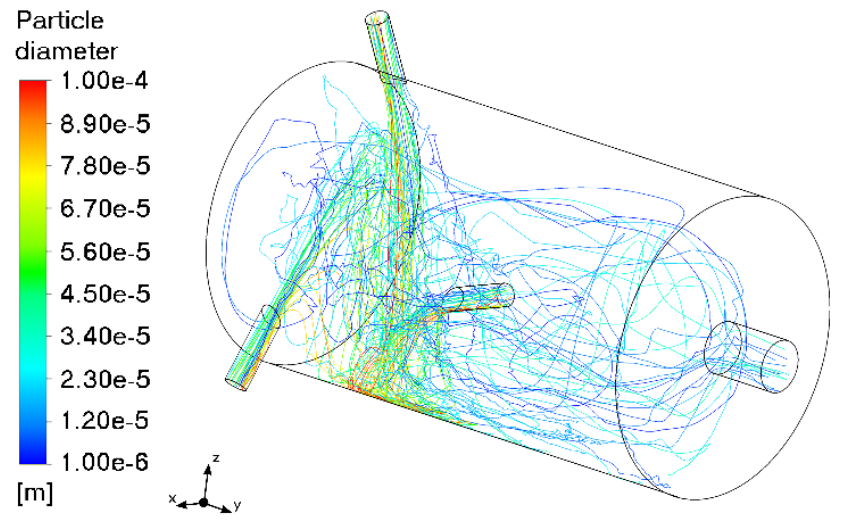

(a)

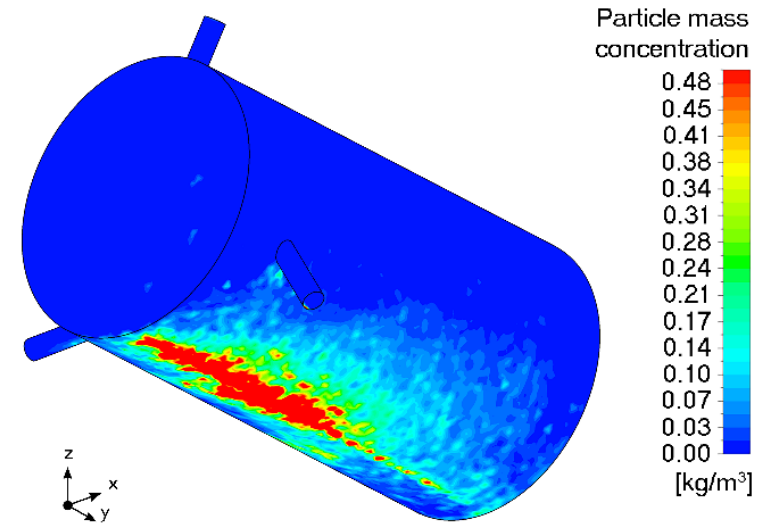

(b)

Figure 9 DPM results for the $1 \mathrm{~kW}$ solar cavity receiver: (a) particle trajectories colored per diameter and (b) particle concentration at the bottom of the cavity.

\subsubsection{Heat transfer enhancement of the fluid domain}

Following on the cold conditions fluid dynamics model, the energy and discrete ordinate radiation model are now accounted for. Extensive elaboration of the optical ray tracing model and its' coupling to the fluid domain has been described elsewhere [27]. The particle cloud in the present study will increase radiative absorption throughout the fluid domain as was previously illustrated in Figure 2. Emissivity and scattering factor of the inert carbon particles are set to 0.5 and 0.9 , respectively. Mass flow rate of the carbon particles that are entrained with the main flow is considered to be of the same order of magnitude as the WIS receiver and thus, is set to a value of $1 \mathrm{mg} / \mathrm{s}$. In parallel with the model presented in Ref. [27], the following boundary conditions are assigned to the fluid domain:

- An inlet velocity of $0.8733 \mathrm{~m} / \mathrm{s}$ is assigned to all three inlet ports which corresponds to an air flow rate of $10 \mathrm{slpm}$ that is used throughout the experimental campaigns.

- Walls of the cavity receiver, which comprise of the cylindrical shell and back plate, were modeled as no-slip walls with conjugated conduction-convection-radiation heat transfer.

- The outlet is defined as a pressure outlet at atmospheric pressure.

- And eventually, the most important boundary condition, i.e. the aperture window, accounts for the irradiation input of the fluid domain. This boundary is located at the interface between the optical model and the CFD domain. As visually shown in Figure 3b the irradiation of the window, which is obtained from an optical Monte Carlo ray-tracing analysis of the solar concentrator, is passed to the fluid domain at the glass solid-fluid interface. Given the large extent of this optical analysis which has been presented in detail in prior publications (i.e. in Ref. [38] and Ref. [27]), details of this boundary condition and optical analysis are not described in the present study for the sake of brevity. 

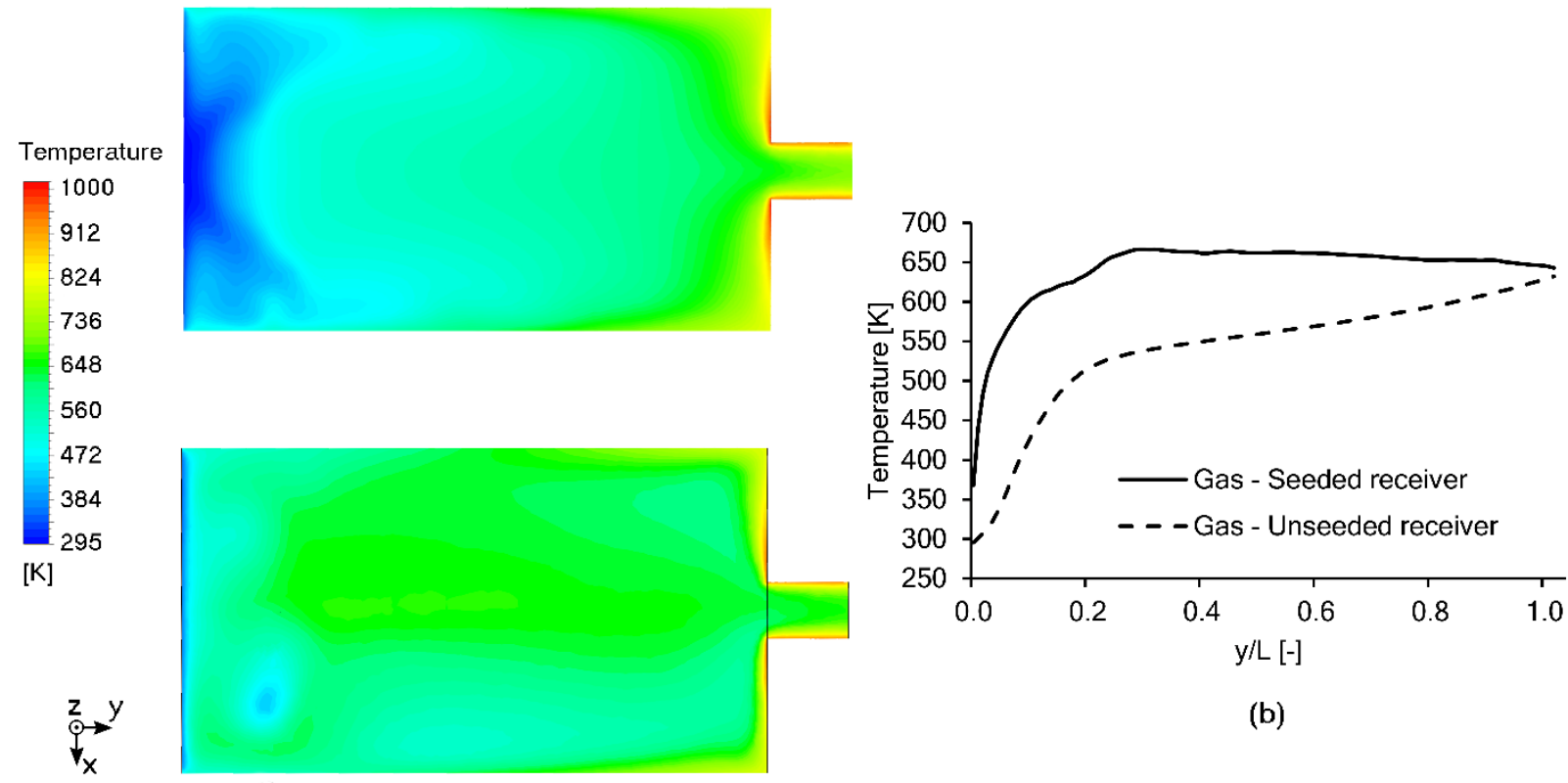

(a)

Figure 10 Temperature results for the unseeded versus the seeded $1 \mathrm{~kW}$ solar cavity receiver: (a) temperature contours in the XY plane and (b) gas temperature distribution along the cavity centerline with respect to the unseeded receiver.

The numerical results in Figure 10 depict the fluid temperature contours in the midplane cross-section of the receiver and the gas temperature distribution, respectively. Compared to the $2 \mathrm{D}$ temperature results for the unseeded receiver, the particle cloud is undoubtedly effective. The colder fluid region at the window has disappeared and the temperature gradient from back to front has diminished. Moreover, Figure 10b corroborates the general assumption of obtaining better temperature uniformity when the receiver is seeded. To quantify the temperature uniformity of the fluid domain a factor is introduced referred to as the Temperature Non-Uniformity or TNU, and equals to

$$
T N U=\frac{\sum|T-\bar{T}|}{n} \times 100
$$

where $T$ is the observed temperature for every position $y, \bar{T}$ is the average temperature over the full range 0 $\leq y \leq L$ and $n$ is total amount of temperature data points observed. A perfectly uniform temperature distribution would result in $\mathrm{TNU}=0 \%$.

The TNU is equal to $19.8 \%$ in case of the unseeded receiver and shows a significant improvement when seeding the receiver, yielding a TNU of $7.3 \%$. Nevertheless, the front part of the cavity close to the window still demonstrates a steep temperature gradient due to the convective losses at the window. The wall temperature distribution is also evaluated but did not show a significant temperature difference with the 
unseeded receiver. Hence, it can be concluded that the particle cloud does not yield that much of a shading effect on the walls. Furthermore, the effect of particle loading should be investigated.

Table 3 Flow parameters for the parametric flow analysis of the seeded $1 \mathrm{~kW}$ cavity receiver.

\begin{tabular}{cccccc}
\hline & \multicolumn{5}{c}{ Case } \\
\cline { 2 - 6 } & $\mathbf{1}$ & $\mathbf{2}$ & $\mathbf{3}$ & $\mathbf{4}$ & $\mathbf{5}$ \\
\hline Flow parameters & & & & & \\
Main flow [L/min] & 10 & 10 & 10 & 5 & 15 \\
Particle feeding $[\mathrm{kg} / \mathrm{s}]$ & $1 \times 10^{-6}$ & $5 \times 10^{-6}$ & $1 \times 10^{-5}$ & $1 \times 10^{-6}$ & $1 \times 10^{-6}$ \\
\hline
\end{tabular}

\subsubsection{Parametric analysis of the flow}

Regardless of the CB deposition at the bottom of the cavity, seeding of the receiver has shown to be promising in terms of enhancing the thermal conditions of the fluid domain. Two controllable parameters allow to modify these conditions, i.e. the flow rate of the gas and the mass loading of the discrete phase. The mass loading here is expressed in terms of mass flow rate $[\mathrm{kg} / \mathrm{s}]$ independently from the main gas flow. In this section the effect of both parameters on the thermal conditions is analyzed. Table 3 shows the set parameters and the temperature results. Case \#1 represents the base case with the settings used in the results above, and is considered as the reference for the other four cases. Comparison of the results showed no significant improvement in terms of reducing particle deposition on the bottom wall. Therefore only temperature results are evaluated in this section.

To evaluate the impact of the parametric changes, average temperatures and the TNU are quantified. In addition to determining the TNU along the centerline of the fluid domain, temperature uniformity is also computed in the full three-dimensional fluid domain. This way it accounts for non-axisymmetric phenomena that would otherwise not be captured by the TNU evaluated along the centerline. Accordingly, the TNU equation from eq. 13 becomes

$$
T N U=\frac{\sum_{i=0}^{N}\left|T_{c e l l, i}-T_{v o l, a v g}\right|}{N} \times 100
$$

where $T_{\text {cell, } i}$ is the gas temperature for cell $i, T_{\text {vol,avg }}$ is the volume averaged gas temperature and $N$ is the total amount of cells inside the computational domain.

\subsubsection{Particle loading}

First, the particle feeding rate is altered with respect to the original value of $1 \times 10-6 \mathrm{~kg} / \mathrm{s}$. With the this value being considered the minimum, increase by a factor of 5 and 10 are set respectively for case \#2 and 
\#3, as seen in Table 3. Increasing the loading of the particles fed with the main flow shows to have a significant impact on the gas temperature. A proportional increase in temperature is observed in the gas temperature distribution in Figure 11a. The averages of these temperatures, i.e. $T_{\text {avg, }, c}$ in Table 4, rise by approximately $130 \mathrm{~K}(20 \%)$ and $200 \mathrm{~K}$ (31\%) for cases \#2 and \#3, respectively. Additionally, a positive enhancement in terms of temperature uniformity along the centerline is observed. However, when considering the complete fluid domain, a less pronounced temperature increase is observed (Table 4). Moreover, the three-dimensional temperature uniformity seems to be adversely affected. The probable reason for these contrasting results can be explained by the increased deposition of the particles when higher particle loadings are used. Figure $11 \mathrm{~b}$ shows several temperature spikes along the bottom cavity wall which are caused by the accumulation of the heavier carbon particles at the bottom of the receiver. This accumulation mainly occurs in the proximity of the inlets and is previously visualized by the particle trajectories in Figure 9a. Thus, increasing the particle loading by a factor of 5 and 10, respectively, has an aggravating effect on the deposition at the bottom of the wall. 

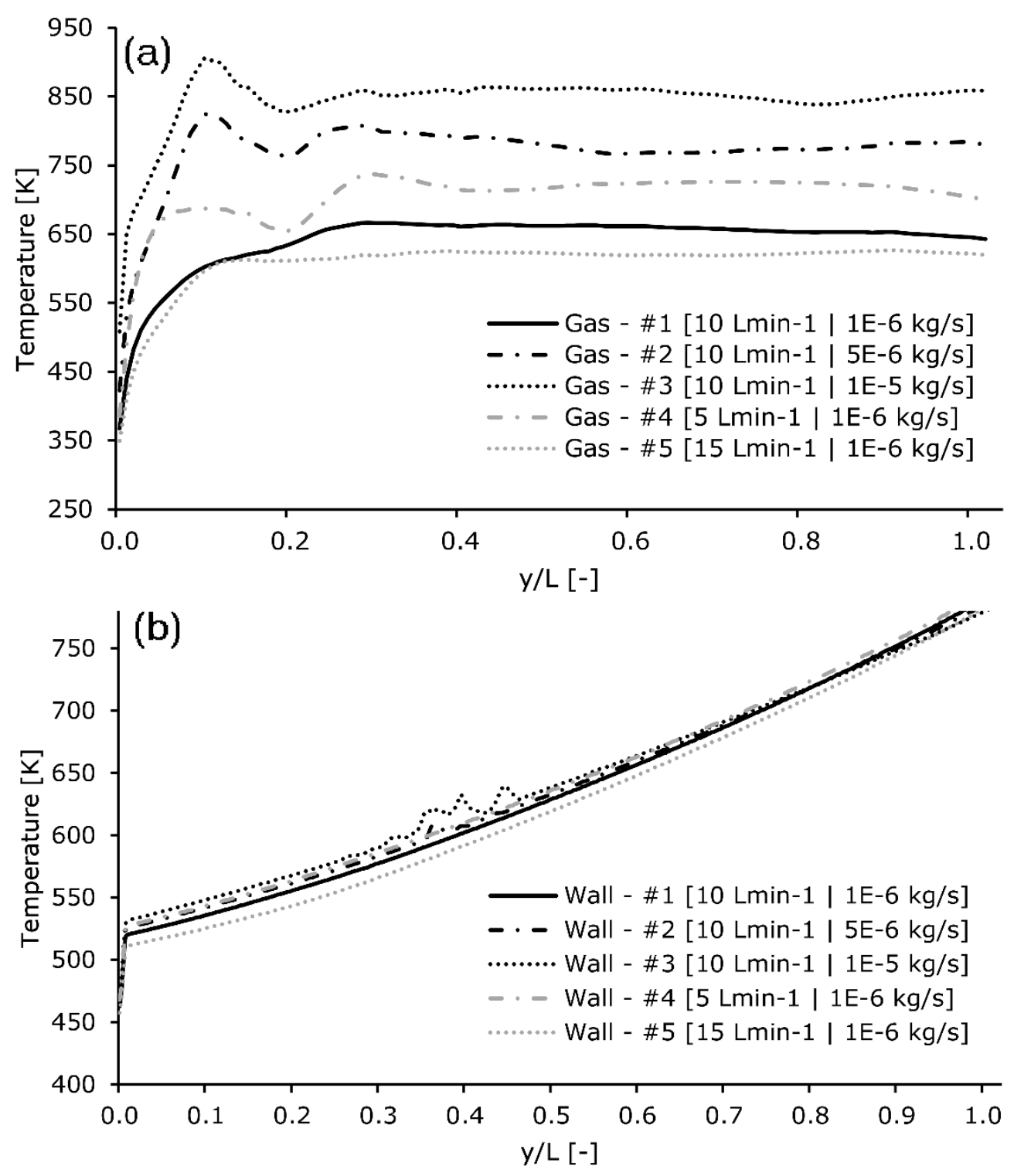

Figure 11 Results of the parametric flow analysis: (a) gas temperature along centerline and (b) wall temperature distribution at bottom of the cavity receiver.

\subsubsection{Flow rate}

In terms of volume flow rate the original flow of $10 \mathrm{~L} / \mathrm{min}$ is both decreased and increased by $50 \%$ while the particle loading is kept the same (Table 3). Intuitively, temperature should increase at a lower flow rate and vice versa. The results in Figure 11 confirm this presumption. The average temperature difference between the reference case $\# 1$ and lower flow rate case $\# 4$ is found to be around $64 \mathrm{~K}$. Increasing the flow rate showed to decrease the gas temperature along the centerline by $32 \mathrm{~K}$ on average. The same applies to the wall temperature, although, at remarkably lower temperature differences. With respect to case \#1 the average differences are $7 \mathrm{~K}$ and $9 \mathrm{~K}$ for cases $\# 4$ and \#5, respectively. Results on the temperature uniformity 
in Table 4 show opposing trends. Considering the centerline distribution, the higher flow rate case \#5 seems to have better temperature uniformity than both cases \#1 and \#4. Conversely, when the temperature distribution throughout the complete fluid domain is considered, the TNU is found to decrease with lower flow rates. The TNU for a higher volumetric flow rate, on the other hand, increases. A lower volumetric flow rate results in higher residence time for both the fluid elements and the discrete phase particles. This positively affects the particle cloud both in terms of diffusive motion of the solid elements, but also in terms of radiative absorption of the particles. One additional conclusion can be drawn based on the average temperature of the gas at the outlet of the receiver, i.e. $T_{\text {avg,outlet }}$ With respect to cases \#2 - \#4, increasing the flowrate had an adverse effect on the temperature. When choosing the appropriate flow rate, careful consideration of its' effect on the temperature should be considered as well as on other aspects (e.g. reaction kinetics).

Table 4 Flow temperature results for the parametric flow analysis of the seeded $1 \mathrm{~kW}$ cavity receiver.

\begin{tabular}{cccccc}
\hline & \multicolumn{5}{c}{ Case } \\
\cline { 2 - 6 } & $\mathbf{1}$ & $\mathbf{2}$ & $\mathbf{3}$ & $\mathbf{4}$ & $\mathbf{5}$ \\
\hline Centerline & 642 & 771 & 846 & 705 & 610 \\
$\mathrm{~T}_{\text {avg,cl }}[\mathrm{K}]$ & 3.6 & 2.6 & 2.2 & 3.3 & 2.9 \\
$\mathrm{TNU}_{\mathrm{cl}}[\%]$ & & & & & \\
Full fluid domain & 620 & 694 & 732 & 675 & 585 \\
$\mathrm{~T}_{\text {avg,3D }}[\mathrm{K}]$ & 9.3 & 10.5 & 11.0 & 8.7 & 10.3 \\
$\mathrm{TNU}_{3 \mathrm{D}}[\%]$ & 770 & 837 & 882 & 838 & 729 \\
$\mathrm{~T}_{\text {avg,outlet }}[\mathrm{K}]$ & & & & & \\
\hline
\end{tabular}

\subsection{Parametric study of the $1 \mathrm{~kW}$ receiver}

As a final stage of the numerical analyses presented in this study, the above-obtained results are utilized to improve upon the initial $1 \mathrm{~kW}$ receiver [27]. Three design iterations are presented and labelled as Solar Cavity Receivers (SCR) SCR-I, SCR-II and SCR-III. The designs are based on the current cylindrical cavity, further on referred to as SCR-0. While geometry and flow inlet/outlet are altered, the same volume of the fluid domain is maintained (i.e. $2.5 \mathrm{~L}$ ) as well as the dimensions of the irradiated window boundary. In this way, residence time of the fluid and solid elements are similar, and the same amount of radiative power enters the cavity. This makes up for a reasonable comparison in terms of flow and temperature. In the proposed designs carbon particles are again fed with the main flow since the prior results showed a considerable improvement on the temperature uniformity. 


\subsubsection{SCR-I}

The first part of the parametric study of the receiver revolves around two aspects: the first one is the ability of the flow to lift and carry the particles downstream. As is previously observed in the DPM results in Figure 9, the discrete solid particles tend to deposit quite rapidly at the bottom of the cavity. Especially the larger and heavier particles, which are dragged down due to gravitation. Moreover, the exhaust position and backplate make it difficult to uplift these heavier particles and carry them downstream. Hence, optimization of the exhaust configuration should be accounted for. In parallel, from a heat transfer perspective, the gas temperature contours in Figure 10a depicted a hotspot in the central region of the backplate. This is caused by the converging radiative beam coming from the solar simulator's ellipsoidal reflector and directly irradiates the backplate. Therefore, the second design improvement aspect accounts for reducing (or eliminating) this hot spot.

The meshed fluid domain of the SCR-I design is shown in Figure 12 with its' dimensional specifications. As can be seen, a conical section is adopted at the backside of the receiver. This conical section is assumed to have a positive effect on the irradiation at the back of the receiver. While still the highest flux densities might be observed on the walls of this conical section, convective heat transfer by the walls to the gas can be enhanced. Not only is the irradiation spread over a larger surface area, but also the tangential momentum of the vortex flow at the wall is increased by the funnel-like section. It can also be seen in Figure 12 that the exhaust is lowered and aligned with the bottom of the cavity. This permits easier entrainment of the particles downstream and prevents the flow from having to uplift the heavier particles. Since the length of the funnel-section is chosen to be $100 \mathrm{~mm}$, the original length of the cylindrical body is shortened from 200 $\mathrm{mm}$ to $180 \mathrm{~mm}$ in order to maintain the same volume. To increase the overall swirl strength of the vortex 


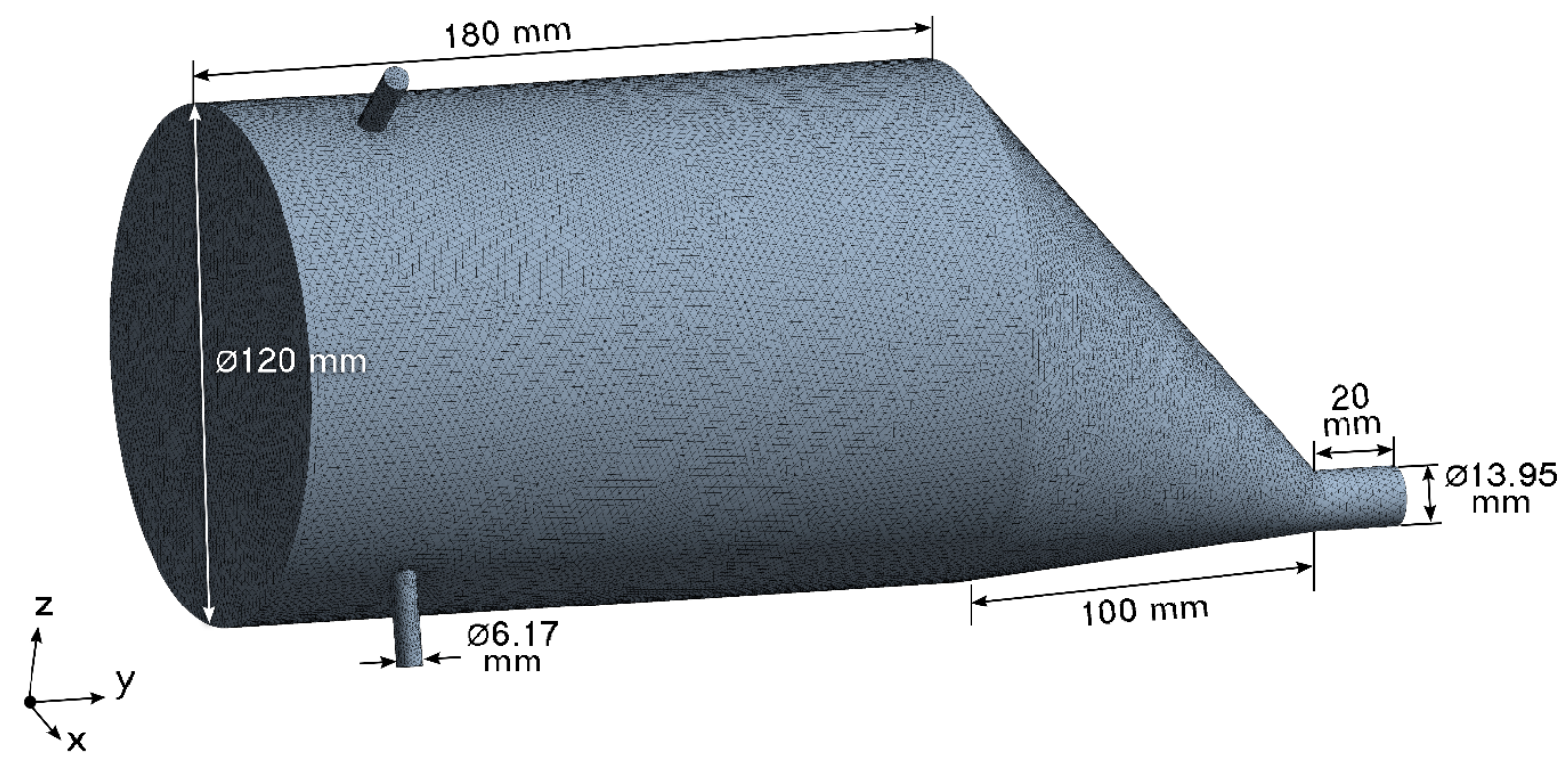

Figure 12 Unstructured mesh and geometric specifications of the optimized receiver design SCR-I (783,540 cells).

from inlet to outlet, the inlet and outlet diameters are decreased by $2 / 3$. Velocities for the boundary condition of the inlet are adjusted accordingly so that the same flow rate of $10 \mathrm{~L} / \mathrm{min}$ is maintained.

Numerical results of the resolved fluid domain are presented in Figure 13. The flow field, shown by the vectors in Figure 13a, seem to depict a similar axi-symmetrical pattern for both receiver geometries. Hence, lowering the exhaust position did not disrupt the flow regime. Lower velocity regions at the intersection of the backplate and shell of SCR-0 are eliminated using the conical section in SCR-I.

Comparison of the temperature is made in terms of gas temperature contours in the YZ-plane (Figure 13b) and the cavity wall temperature (Figure 13c). Although improvement of the receiver design aimed at reducing or eliminating the hot spot on the backplate, the temperature distribution on the frustum is significantly higher for SCR-I. It is evident from these observations that a big portion of the incident converging radiative beam on the backplate in SCR-0 propagates into the exhaust section. This, opposed to the beam directly irradiating the frustum in SCR-I. Furthermore, the weak turbulence of the internal quasitransparent flow along the conical wall is unable to carry away the available heat as seen in Figure $13 \mathrm{~b}$. However, the flow along the conical wall yielded an increase of the exhaust gas temperature from $770 \mathrm{~K}$ to $838 \mathrm{~K}$. In a similar way, the volume averaged temperature of the fluid domain increased from $620 \mathrm{~K}$ to 672 K. As expected for the temperature uniformity in the complete fluid domain, a slightly higher TNU is found yielding an increase from $9.3 \%$ (SCR-0) to $10.0 \%$ (SCR-I). 
(a)
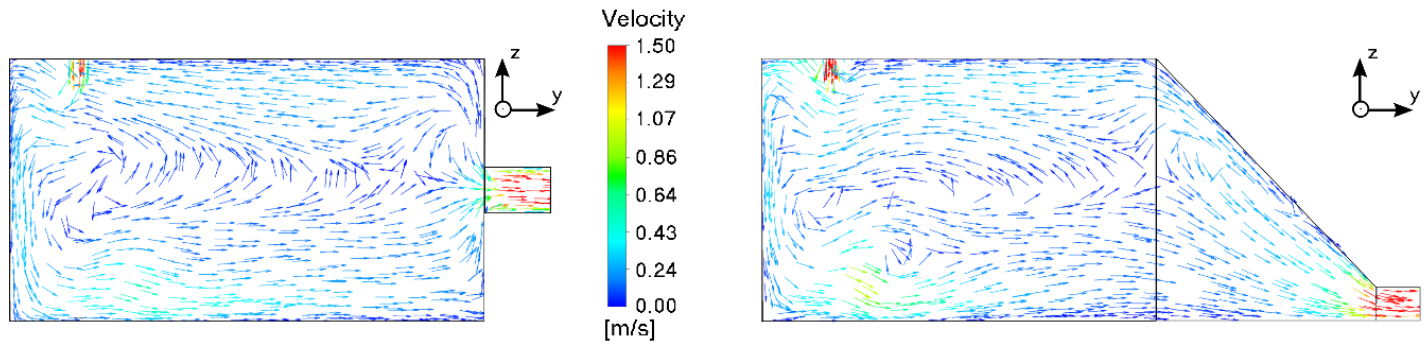

(b)

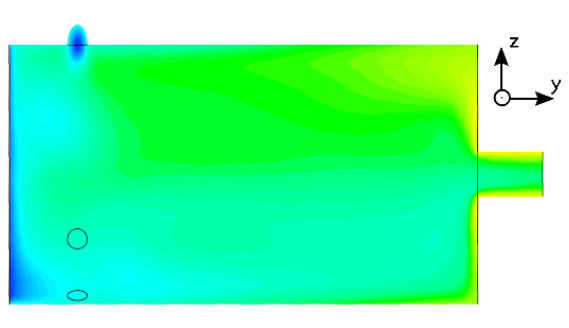

Temperature

1094
994
-894
794
695
595
495
395
296
$[\mathrm{~K}]$

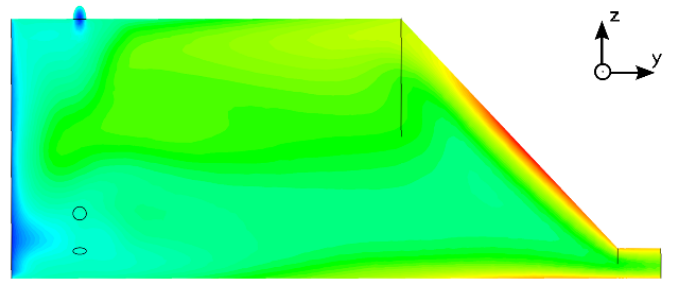

\section{๑)}
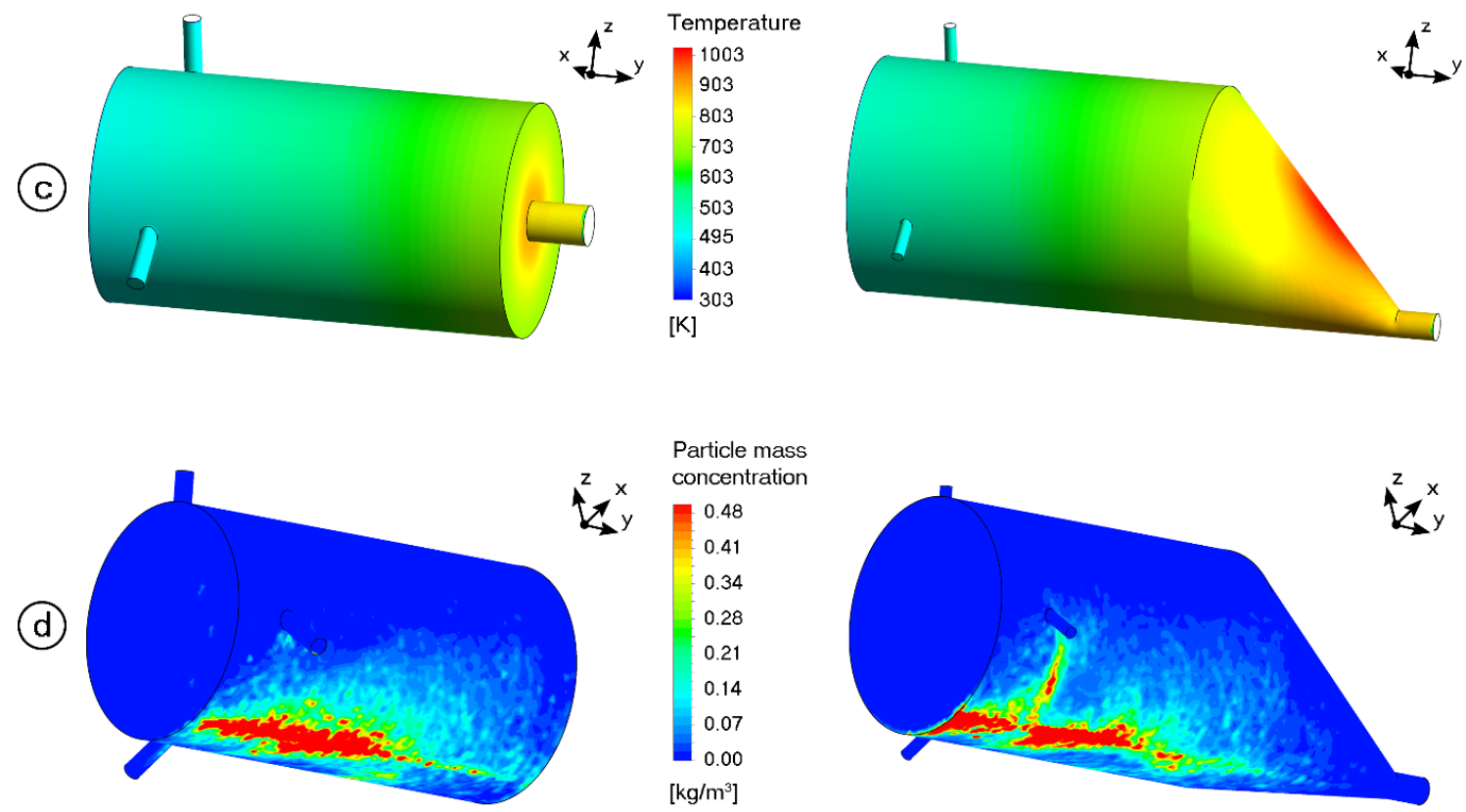

Particle mass concentration

0.48
0.41
0.34
0.28
0.21
0.14
0.07
0.00
$\left[\mathrm{~kg} / \mathrm{m}^{3}\right]$

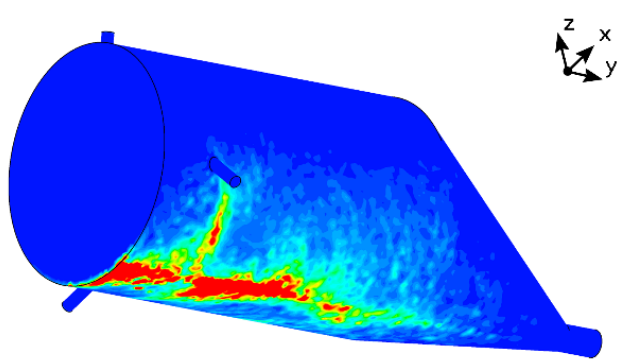

Figure 13 Numerical simulation results for SCR-I (right) with respect to the initial receiver design SCR-0 (left): (a) velocity vector field, (b) temperature contours, (c) wall temperature and (d) particle mass concentration along the cavity walls.

The results from tracking the discrete phase elements (i.e. particles) are visualized through particle mass concentration in the cells adjacent to the cavity wall in Figure 13d. At first sight, no improvement on reducing the amount of deposited carbon is determined in SCR-I. The reduction of the inlet diameter yielded an intended increase of momentum which is visible in Figure 13d: the increased inlet velocity allows the flow to entrain particles up the wall. Nevertheless, the particles are still too heavy for the chosen flow 
conditions to carry them downstream. Observation of the concentration at the window shows little deposition at the bottom of the window in receiver SCR-I, which is not observed in receiver SCR-0.

Alternatives should be considered independent from the cavity design and/or flow configuration to address this particle deposition. This leads, for example, to re-evaluating the particle size distribution assigned to the solid elements in the numerical model. The previously used Rosin-Rammler distribution is fitted to particle size data of Fluka 03866 from Hirsch and Steinfeld [34]. The distribution considers a mean particle diameter of $d_{p, m}=20 \mu \mathrm{m}$ and a spread parameter $n$ of 1.2. In literature, several studies have advocated the use of submicron particle sizes for solar thermal(-chemical) applications such as in Ref. [24] and Ref. [33]. This does not only allow for better suspension in the main flow, but also improves overall radiation absorption of the medium. Therefore, a new particle size distribution is considered in the numerical model. A Rosin-Rammler distribution is fitted to the size data of the carbon powder (Asbury No. 5358) used by Klein et al. in their particle seeding experiments [39]. A Matlab ${ }^{\circledR}$ tool specifically tailored for RosinRammler fitting [40] yielded the parameters of the distribution, i.e. $d_{p, m}=7.16 \mu \mathrm{m}$ and $n=0.759$. The same particle size range of 1 to $100 \mu \mathrm{m}$ for the carbon particle feed is maintained in the numerical model.

Effect of the new particle size distribution on the gas temperature throughout the fluid domain is shown in Figure 14. Comparison is made for both receivers SCR-0 and SCR-I. Figure 14a represents the receivers adopting the coarse size distribution while Figure $14 \mathrm{~b}$ shows the temperature results for the finer particle size ditribution. It can be seen that temperature has slightly increased, especially in the upper half of the fluid domain. The contour shapes remain the same while only the temperature values are amplified when finer particles are used. While the volume-averaged temperature has further increased by $85 \mathrm{~K}$ and $75 \mathrm{~K}$ for the SCR-0 and SCR-I design, respectively, the finer particle suspension deteriorates temperature uniformity with TNU increasing approximately $0.5 \%$.

By adopting a finer size distribution less particle deposition is observed at the bottom of the receiver. This is shown in Figure 15, which plots the particle mass concentration at the bottom of the receiver in the longitudinal direction. Comparison between both receivers SCR-0 and SCR-I is made using both aforementioned particle size distributions respectively. The overall trend observed in all four distributions depicts the highest concentration to be next to the inlet ports around $y / L=0.2$. With the inlet ports being positioned at $y / L=0.15$, the effect of increased momentum at the inlets of SCR-I reduces the carbon particle 


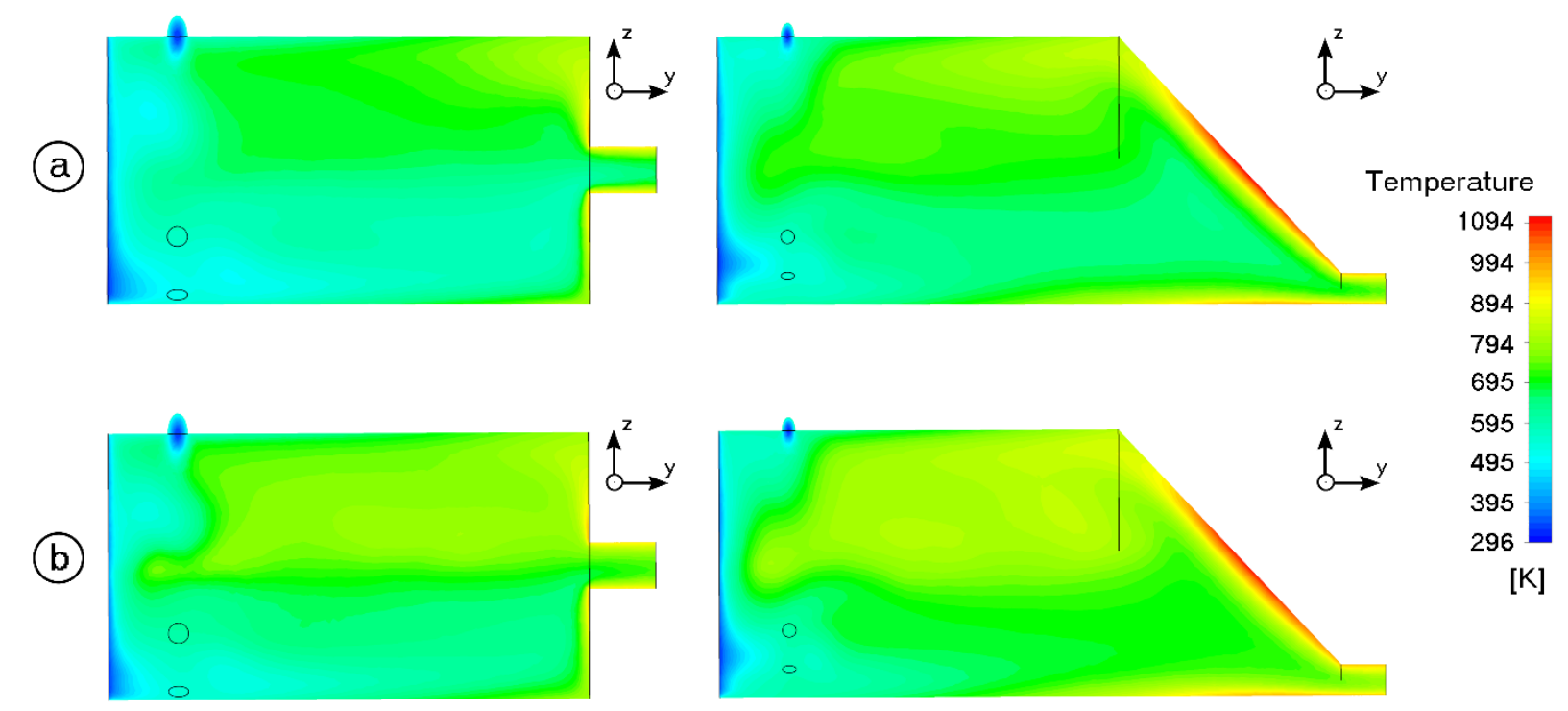

Figure 14 Temperature contours in the YZ-plane for both receivers SCR-0 and SCR-I: (a) Rosin-Rammler distribution with $d_{p, m}=$ $20 \mu \mathrm{m}$ and spread parameter $n=1.2$, and (b) Rosin-Rammler distribution with $d_{p, m}=7.16 \mu \mathrm{m}$ and $n=0.759$.

deposition at this axial location. Using smaller particle sizes, i.e. 'SCR-0 - Fine' and 'SCR-I - Fine' in Figure 15, clearly results in less deposition at the bottom of the cavity. The average particle mass concentration along the whole cavity wall reduces from $0.0444 \mathrm{~kg} / \mathrm{m}^{3}$ to $0.0177 \mathrm{~kg} / \mathrm{m}^{3}$ for SCR- 0 and from $0.0452 \mathrm{~kg} / \mathrm{m}^{3}$ to $0.0169 \mathrm{~kg} / \mathrm{m}^{3}$ for SCR-I.

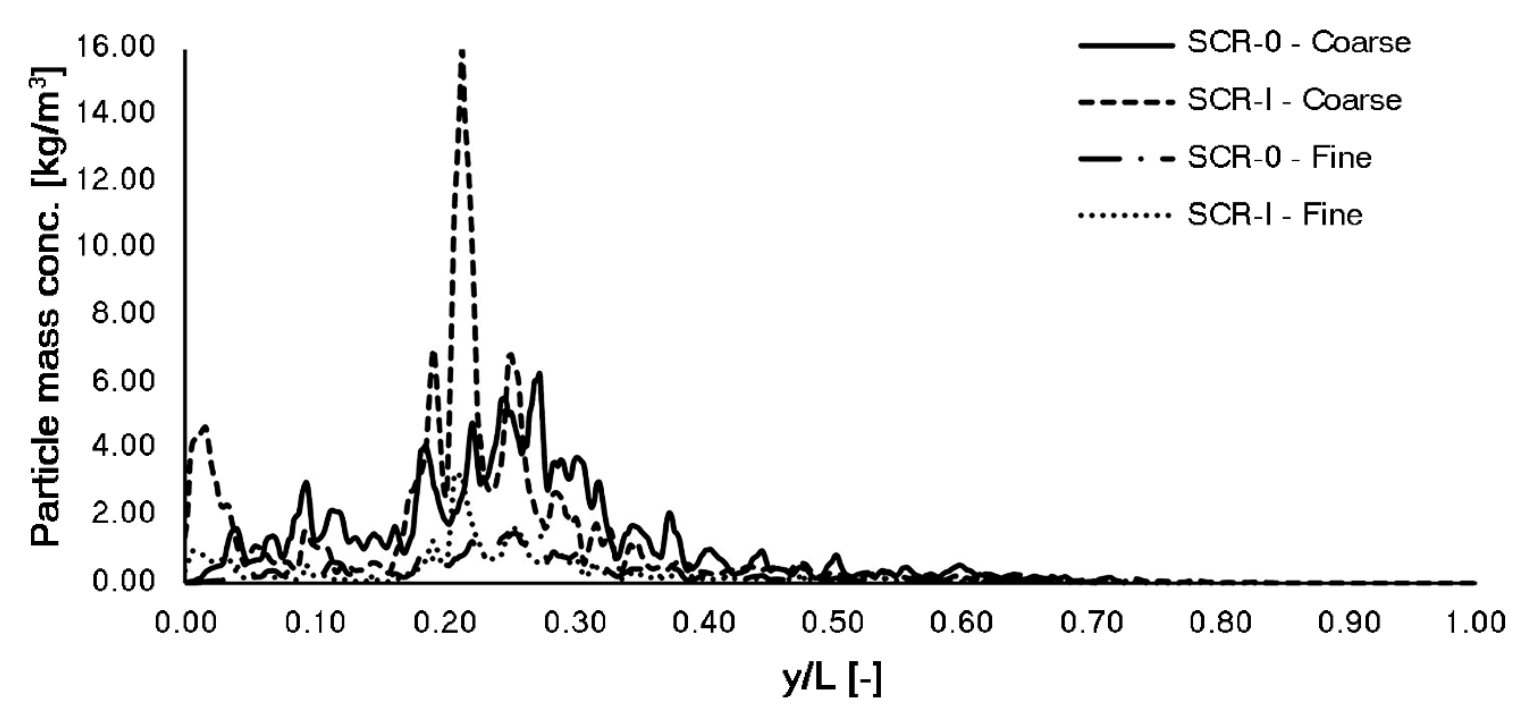

Figure 15 Particle mass concentration distribution along the bottom of the receiver for SCR-0 and SCR-I and for both particle size distributions. 


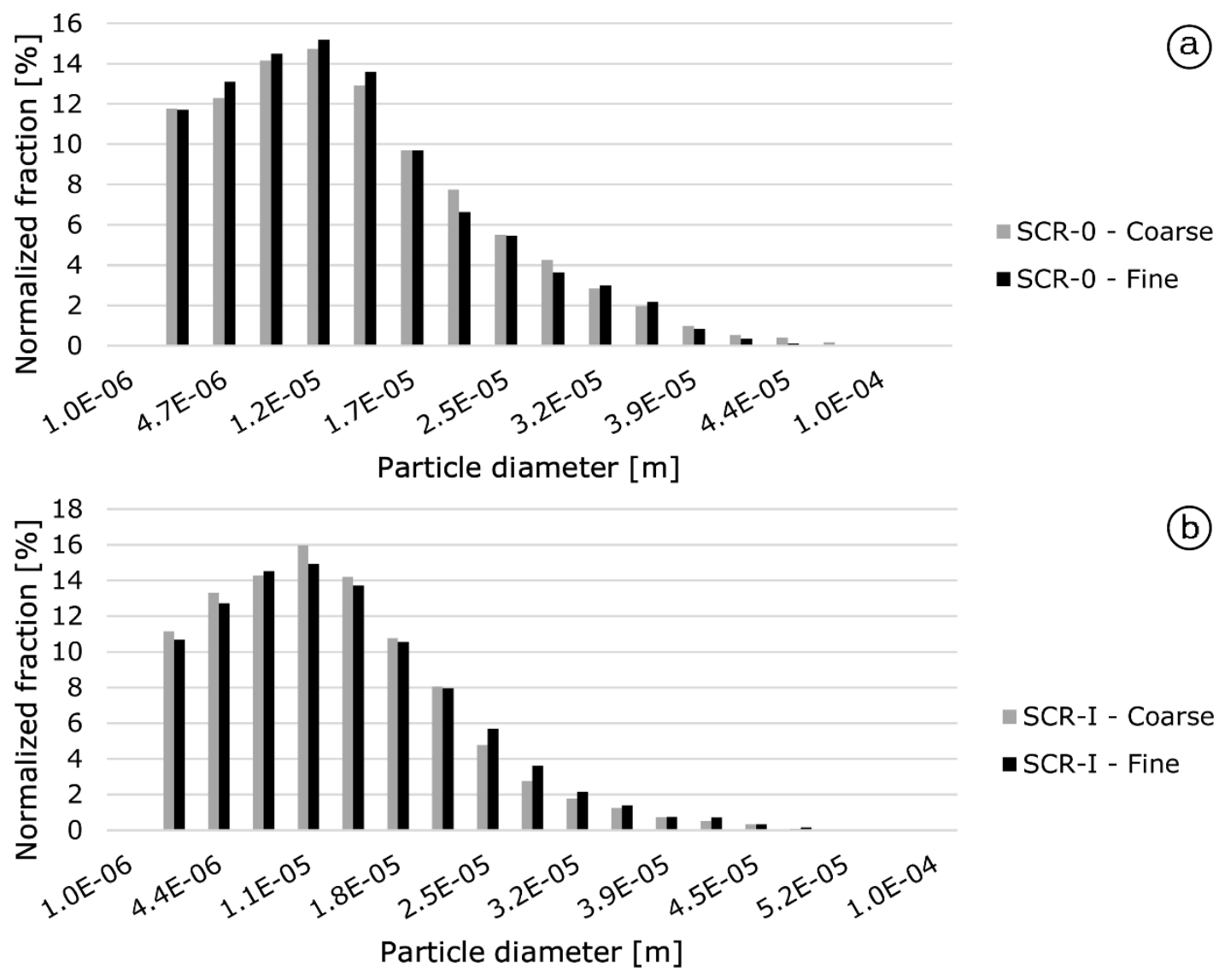

Figure 16 Particle size distributions at the receiver outlet for (a) SCR-0 and (b) SCR-I.

A final comparison for the above four cases is made to evaluate the range of particle sizes over which the flow is able to carry the particles downstream. Figure 16 shows two histogram plots of both the coarse and fine particle size distributions, which is tracked and computed at the outlet for both receiver designs. The normalized fraction is computed based on the counted number of particles per diameter range reaching the outlet of the receiver and is normalized over the total count. The comparison of the coarse distributions for both receivers, i.e. gray bars, shows that the distribution is slightly more skewed towards smaller particle diameters in receiver SCR-I. Furthermore, the histograms reveal that using a finer particle size distribution for the seeded carbon black leads to relatively more particles with larger diameters reaching the exhaust of the SCR-I receiver. Hence, this might confirm that lowering the exhaust port positively affects the entrainment of larger particles out of the cavity. However, with respect to SCR-0, Figure 16 does not conclusively identify receiver SCR-I to be superior in terms of particle entrainment.

\subsubsection{SCR-II}

Based on the findings and observations in the comparative assessment of SCR-0 and SCR-I, a new receiver design is proposed. The fluid domain of this receiver (SCR-II) is presented in Figure 17. The SCR-I 
geometry is considered as the basis on which improvements were implemented. The cylindrical front-body is shortened to a length of $90 \mathrm{~mm}$ opposed to the conical frustum that is elongated to a length of $320 \mathrm{~mm}$. From the observed hot spot in Figure 13c and with the initial goal of the frustum to spread the incident radiative flux over a larger surface area, adopting a significantly longer conical backpart is presumed to enhance heat transfer in the back of the receiver. Moreover, the fluid flow is assumed to benefit from this modification in terms of convectively transferring heat away from the walls. The overall volume of the receiver is again kept the same (i.e. $2.5 \mathrm{~L}$ ), and so are the in- and outlet diameters. The carbon seeding in the model adopts the same fine distribution as is used in the latter section.

Although the complexity of the geometry is relatively low, manufacturability of the design is neglected at this stage. Two further modifications of the flow configurations are implemented. The first one applies to the three tangential inlet ports. Instead of only considering tangentiality of the inlets in one plane, the ports are pivoted by $30^{\circ}$ towards the back of the receiver. This modification allows to eject and spread the particle suspension further downstream and, thus, preventing deposition on the window.

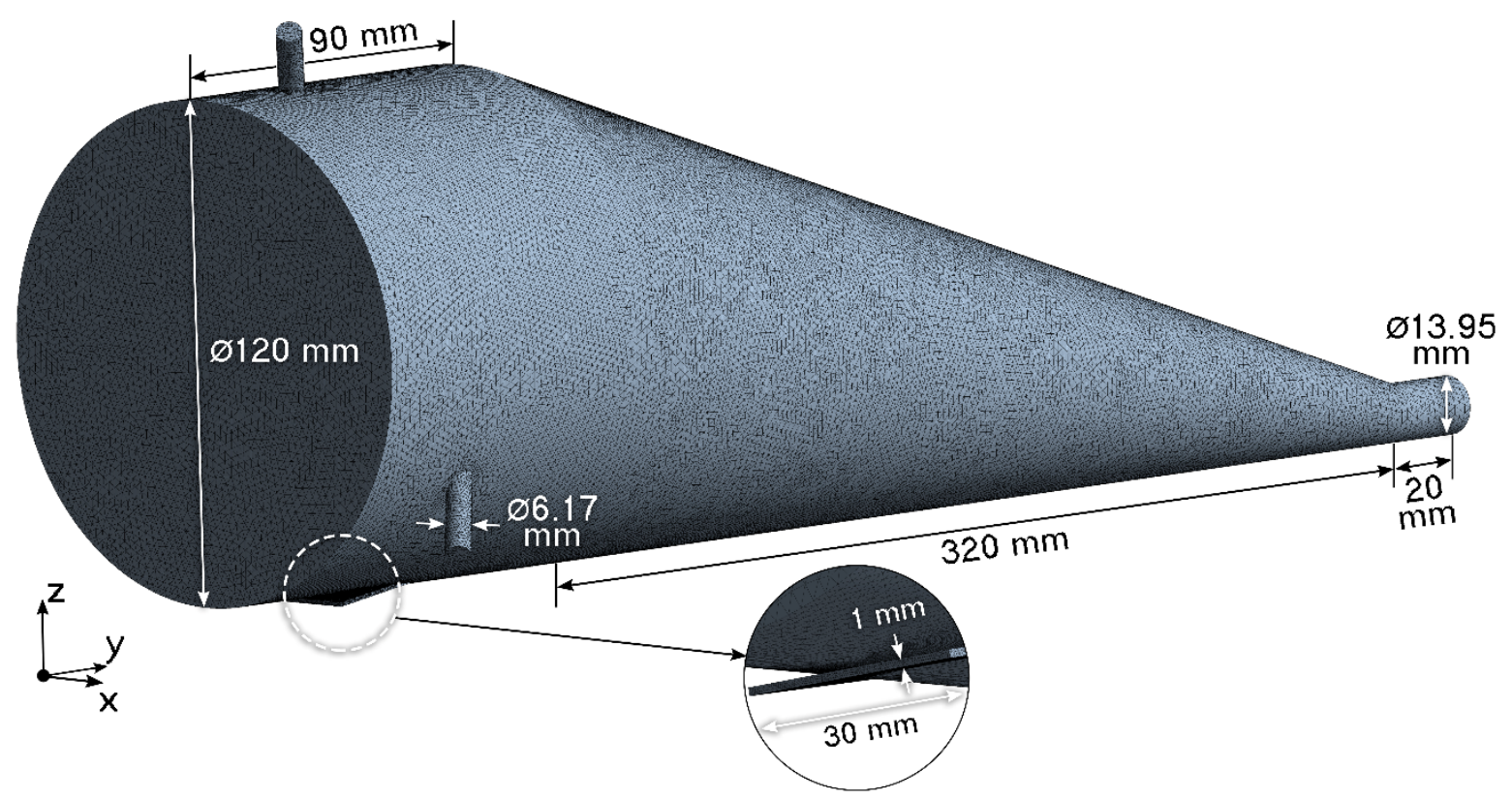

Figure 17 Unstructured mesh and geometric specifications of the optimized receiver design SCR-II (940,474 cells).

A possible drawback of this flow modification might be the weaker formation of the vortex structure as the tangential velocity is decreased at the inlets. However, this might be compensated for by the frustum. A numerical study on the comparison between a cylinder-cone tube (similar to the SCR-II geometry) and a cylindrical swirl tube, to be used as centrifugal separators, showed that tangential velocity inside a 
cylindrical swirl tube tends to decrease as the flow progresses downstream [41]. The second design modification involves the addition of a wall screening flow. In contrast with the wall screening flow of the WIS receiver [17] presented in section 3.1, the inlet proposed in SCR-II does not circumscribe the receiver. Instead, this flow is aimed at preventing particle deposition at the bottom of the chamber and carrying the heavier particles downstream towards the exhaust. Hence, a slot-type inlet at an angle of $8^{\circ}$ with the bottom wall is added to the receiver, as seen in Figure 17. A slot width of $30 \mathrm{~mm}$ is chosen to sufficiently cover the bottom wall based on the earlier DPM results. Thickness of the slot is dimensioned at $1 \mathrm{~mm}$ which should allow for a sufficiently high inlet velocity, in order for the screening flow to be effective. An arbitrary ratio of the $80 \% / 20 \%$ is applied to the flow rates. The original main flow rate of $10 \mathrm{~L} / \mathrm{min}$ is lowered to $8 \mathrm{~L} / \mathrm{min}$ while a volumetric flow rate of $2 \mathrm{~L} / \mathrm{min}$ is assigned to the screening flow. Also air is used for the wall screening flow. Numerical solver settings and other parameters were taken the same as in the SCR-I computation.

The obtained results for the numerical computation of the SCR-II fluid domain are shown in Figure 18. The velocity vector field in Figure 18a indicates that with respect to the initial strong vortex core that was originally present in SCR-0 [27], the flow pattern has transitioned into a plug-like flow. This is mainly observed in the frustum as the flow approaches the exhaust. This is also reflected in the temperature contours in Figure 18b where a colder fluid zone is observed at the lower half of the cavity. This is due to the combined effect of weak swirl strength, which affects the flow mixing, and buoyancy of the hotter gas. Although the effect of the screening flow on this cold gas region could be questioned, a similar temperature contour pattern is observed in SCR-0 and SCR-I (Figure 14). Hence, the effect of wall screening on the temperature distribution is neglected.

As can be seen in the wall temperature distribution in Figure 18c, elongation of the frustum for the SCR-II design has positively impacted the temperature distribution. The incident radiation originating from the solar simulator and irradiating the back wall of the cavity is spread over a larger surface area. The maximum incident radiative flux on the wall is decreased by approximately $100 \mathrm{~kW} / \mathrm{m}^{2}$ in SCR-II with respect to the maximum flux in SCR-I. This leads to a maximum wall temperature decrease from $1100 \mathrm{~K}$ (SCR-I) to 1023 K (SCR-II). The particle concentration in Figure 18d shows a significant reduction with respect to the DPM results of SCR-0 and SCR-I in Figure 13d. With respect to the average concentration on the cavity 
(a)

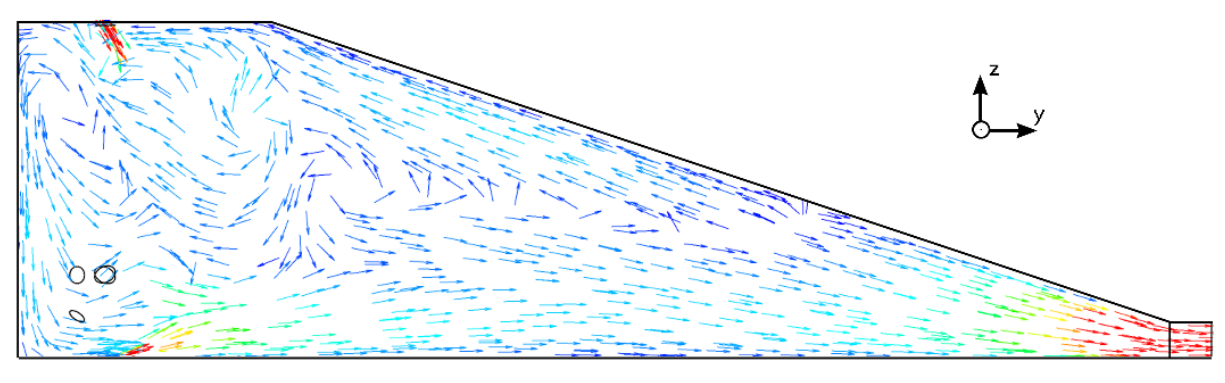

(b)

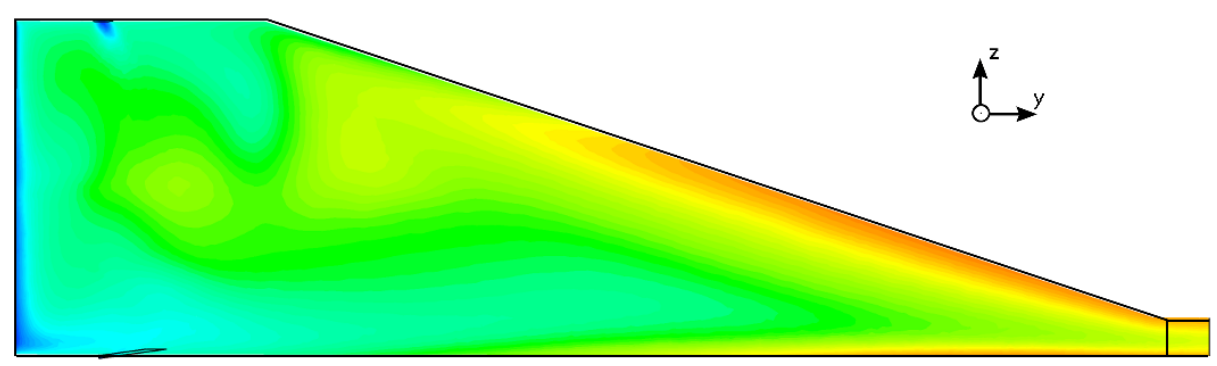

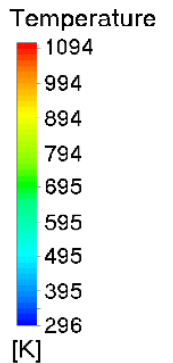

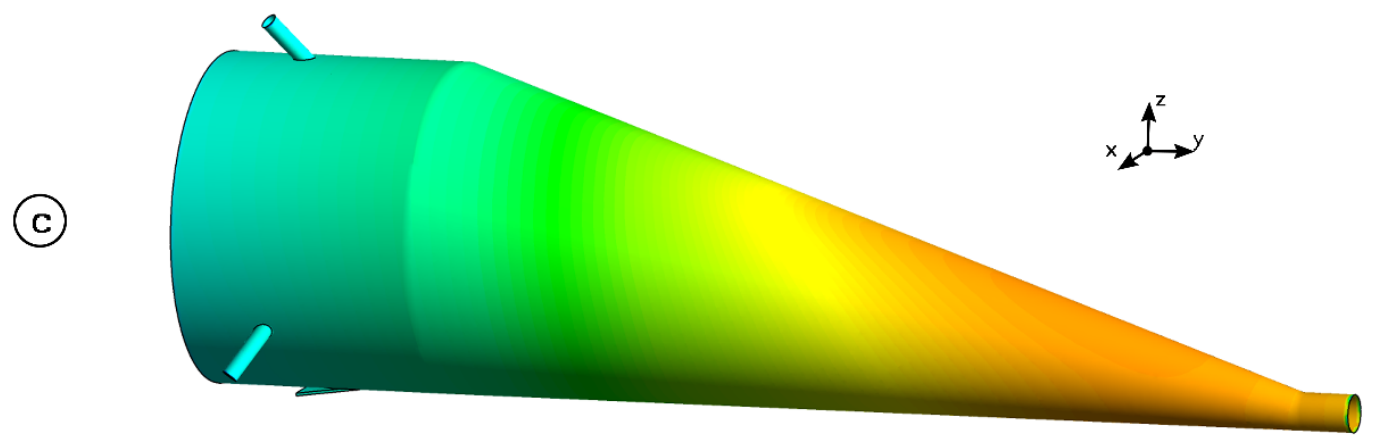

Temperature

\begin{tabular}{l}
\hline 1003 \\
-903 \\
803 \\
703 \\
603 \\
-503 \\
495 \\
403 \\
303
\end{tabular}

$[\mathrm{K}]$

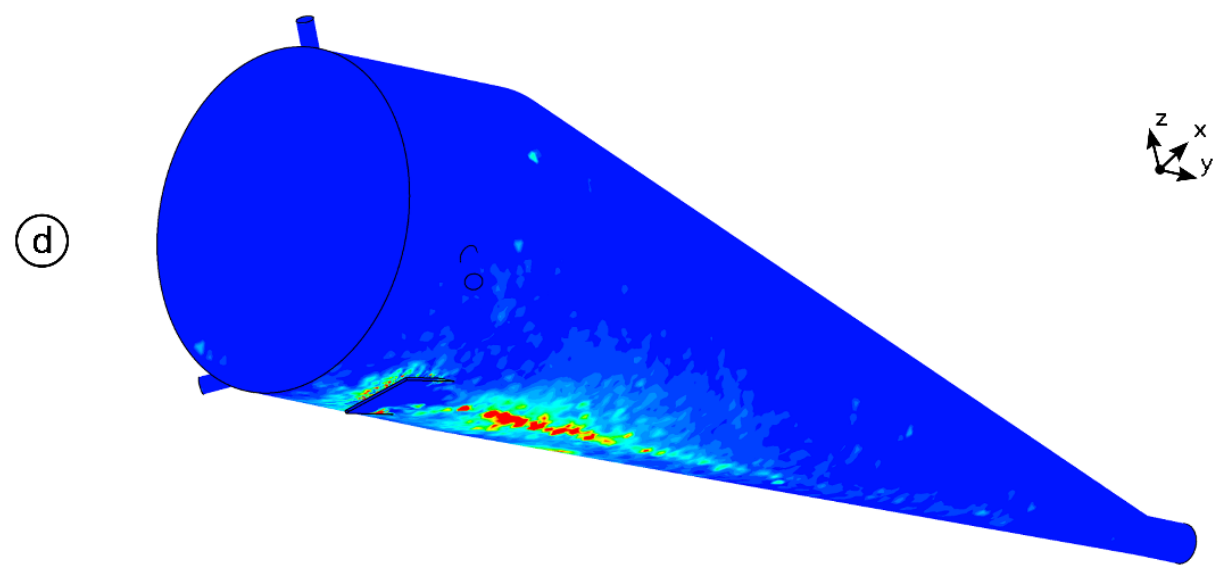

Particle mass concentration

0.48
0.41
0.34
0.28
0.21
0.14
0.07
0.00
$\left[\mathrm{~kg} / \mathrm{m}^{3}\right]$

Figure 18 Numerical simulation results for SCR-II: (a) velocity vector field, (b) temperature contours, (c) wall temperature and (d) particle mass concentration along the cavity walls. 


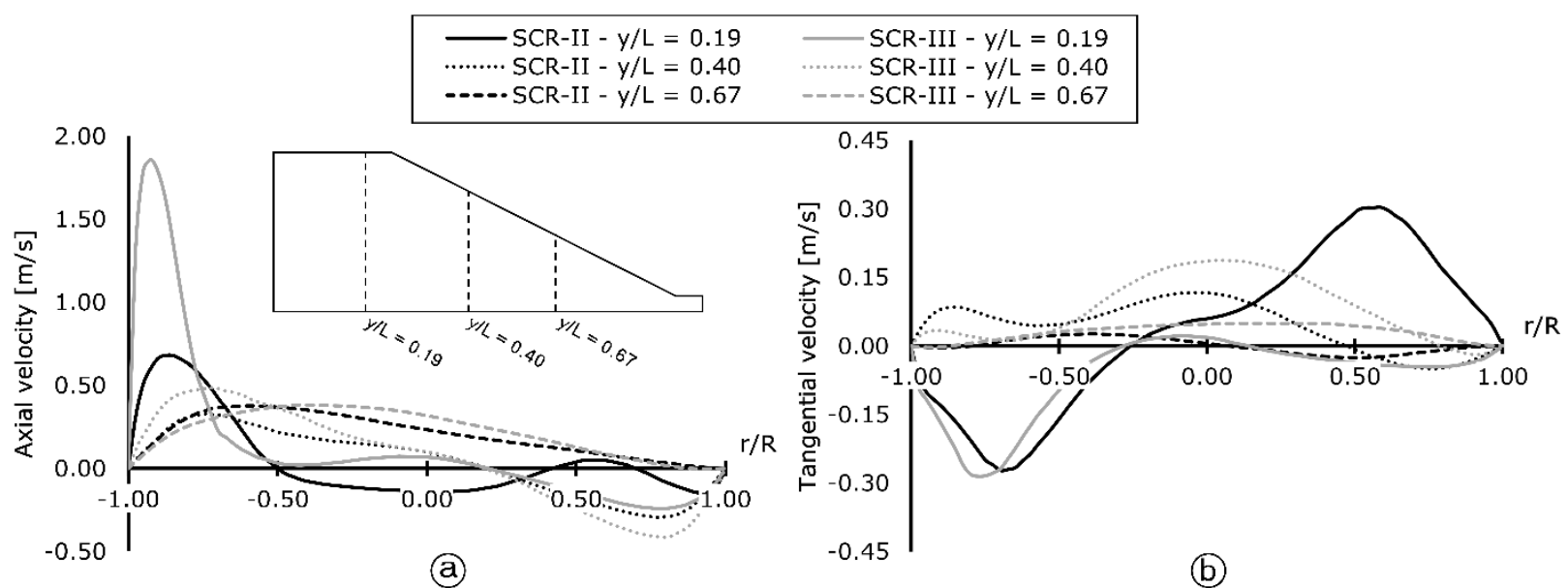

Figure 19 (a) Axial velocity distribution and (b) tangential velocity distribution for SCR-II and SCR-III.

walls of $0.0167 \mathrm{~kg} / \mathrm{m}^{3}$ in SCR-I, the value has decreased to an average concentration of $0.0142 \mathrm{~kg} / \mathrm{m}^{3}$ in SCR-II. Nevertheless, the latter design allows room for improvement.

\subsubsection{SCR-III}

In the previous design it is assumed that pivoting the inlet ports towards the back of the receiver would have an attenuating effect on the swirl strength. In addition, since deposition of the particles is still present on the bottom wall of the cavity, the effect of altering the wall screening flow should be investigated. Hence, a final design iteration is applied leading to the SCR-III design. The geometry is identical to the SCR-II in Figure 17 with the exception of the flow inlet ports. All three tangential inlets are re-configured as before, i.e. in one and the same plane at an angle of $30^{\circ}$ with the surface normal where the inlets connect to the cavity. The wall-screening slot is reduced to a size of $20 \mathrm{~mm}$ by $0.5 \mathrm{~mm}$. The same flow rate is maintained which results in a higher inlet velocity.

First a comparison is made with the flow velocities in SCR-II. The axial and tangential velocity profiles are shown in Figure 19a and b, respectively. These distributions are assessed at three different locations along the cavity length to evaluate the cyclone structure of the flow. From the axial velocity profiles one can clearly notice the effect of the screening flow at the bottom of the cavity. Decreasing the slot size while maintaining a same flow rate in SCR-III led to an amplification with a factor of 3 for the peak axial velocity at $y / L=0.19$, with respect to the profile of SCR-II. As the flow progresses downstream, at $y / L=0.67$ the axial flow velocity distributions become skewed towards the bottom of the receiver. While the presence of the screening flow mainly affects the distribution upstream, the skewed distribution at $y / L=0.67$ is caused by the oblique cone shape of the frustum. As a result, the axi-symmetry of the flow and strong vortex structure, which is previously present in SCR-0, is diminished in SCR-II and SCR-III. This is also supported by the tangential velocity profiles. At $y / L=0.19$ the typical sine-wave pattern, indicating the formation of 
a vortex structure, is only observed for the SCR-II design. This is in contrast with the presumption that the pivoted main inlet ports in SCR-II would negatively affect the vortex-generating swirl. Nevertheless, the tangential velocity profiles downstream indicate the absence of a vortex-like fluid motion.

The effectiveness of the changes made in the SCR-II design in terms of preventing particle deposition is visualized in Figure 20. Comparing the particle concentration contours on the walls of SCR-III (Figure 20a) with the prior results for SCR-II (Figure 18d) the increased velocity of the wall screening inlet is able to displace the concentrated deposition slightly further downstream.

(a)
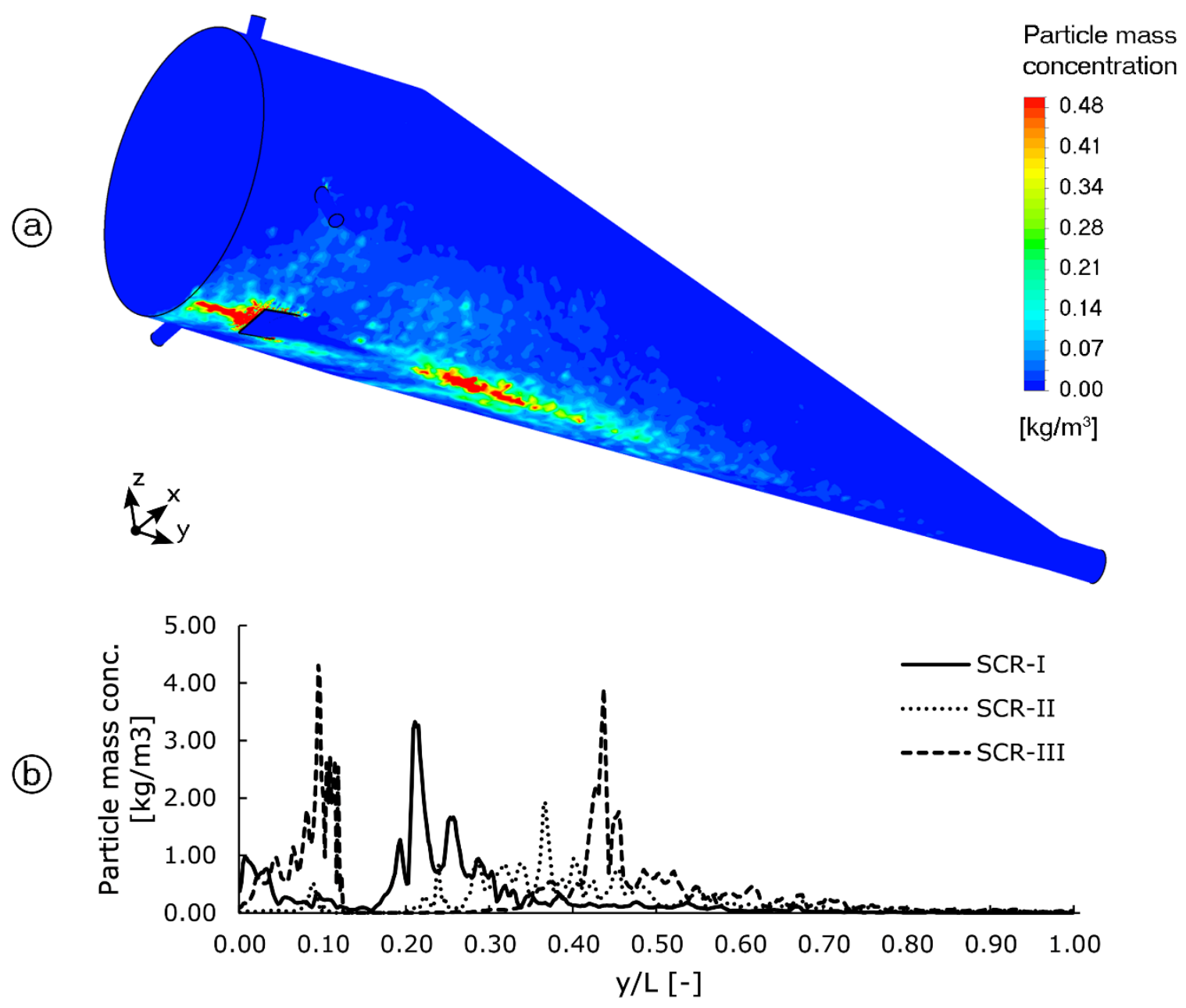

Figure 20 Particle mass concentration for SCR-III: (a) contours on the cavity wall, and (b) concentration distribution at the bottom of the cavity.

However, re-aligning the main inlet ports in the same plane for SCR-III leads to more solid elements in the flow region between the window and the inlets. This eventually leads to a larger concentration of deposition between the screening inlet and the cavity window. This is also clearly visible in Figure $20 \mathrm{~b}$ for $0<y / L<$ 
0.12 along the bottom wall. Moreover, from this figure it can be seen that the flow configuration of SCRII is able to effectively spread the concentration from SCR-0 further downstream.

Final comparison of the optimized designs is made in terms of the particle size distributions at the outlet of the cavity. Figure 21 shows a histogram with the relative fractions of the particle sizes at the exhaust of the cavity receiver. The histogram bins are normalized over the total amount of particles and show the proportional amount of particles for a given particle diameter. The comparison is made for all three design iterations of SCR-0. While all three distributions follow a similar trend, as a result of the R-R distribution at the inlet, the SCR-II distribution shows a clear deviation with the other two distributions: relatively to SCR-I and SCR-III, the particles at the exhaust of the SCR-II receiver are generally larger. The weighted average diameters are respectively $\bar{d}_{S C R-I}=14.8 \mu \mathrm{m}, \bar{d}_{S C R-I I}=16.0 \mu \mathrm{m}$ and $\bar{d}_{S C R-I I I}=14.5 \mu \mathrm{m}$. This is presumably a result of the main inlet ports that were pivoted towards the back of the receiver. It can therefore be concluded that the flow configuration in SCR-II does not only prevent deposition at the front of the cavity (unlike SCR-III), but also is able to carry away heavier particles downstream out of the considered fluid domain.

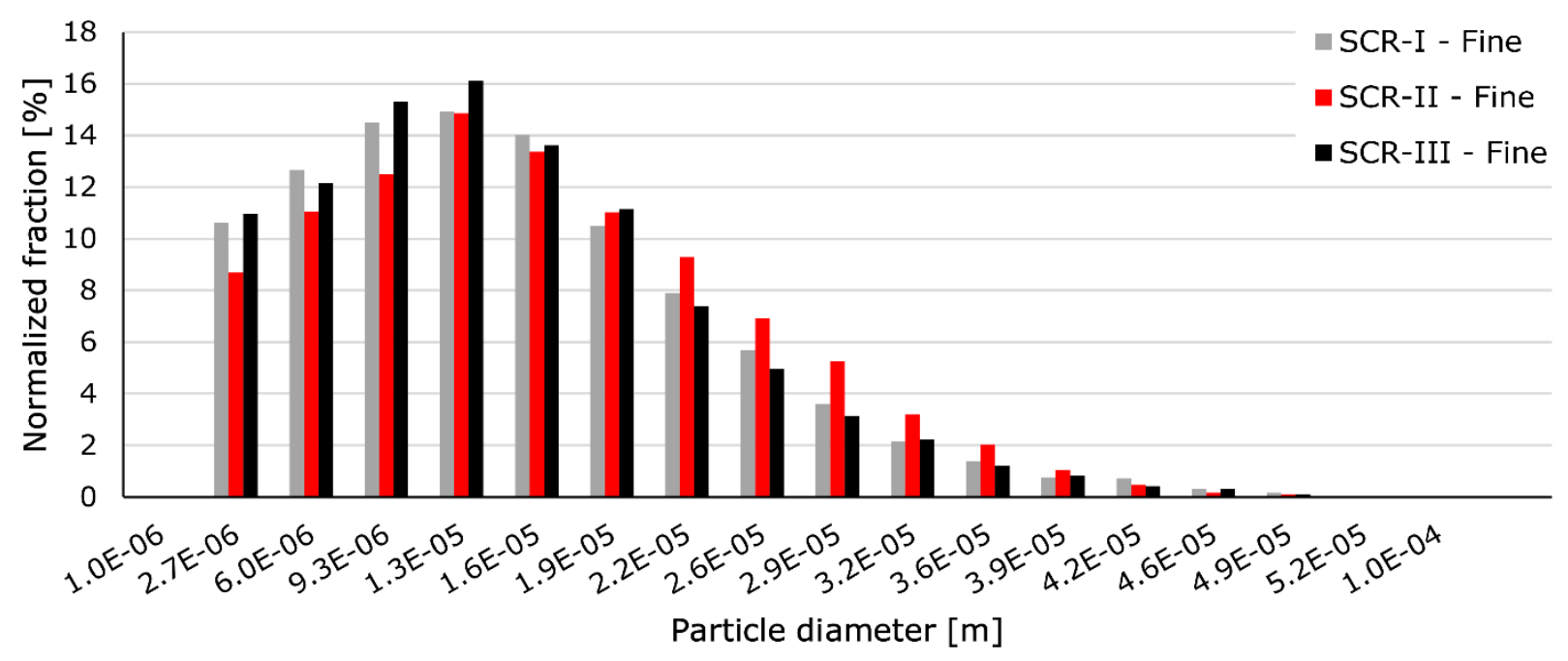

Figure 21 Particle size distribution at the outlet of the cavity receiver for SCR-I, SCR-II and SCR-III.

In conclusion, a quantitative overview of the temperature and DPM results is shown in Table 5. Although temperature uniformity throughout the fluid domain has not been improved in the parametric design iterations, the average gas temperature has been increased. While these metrics are affected by the direct irradiation of the wall in the back of the receiver, alteration of the receiver geometry through elongation of the frustum has effectively spread the radiative flux leading to a maximum wall temperature decrease of at 
least $70 \mathrm{~K}$. The lower maximum wall temperature for the original receiver (SCR-0) is due to the fact that the exhaust is positioned there where the incident flux would be highest. One other important observation is the outlet gas temperature. This temperature reflects the ability of the flow medium to absorb the available heat in the chamber. Higher values can lead to higher production yields when chemically reacting flows are involved (e.g. methane cracking). Hence, the improvements applied to SCR-II and SCR-II have positively affected the exhaust gas temperature and should therefore be considered for solar reactor designs. And lastly, the average particle mass concentration on the walls $c_{\text {avg,walls }}$ supports the DPM results shown before. The SCR-II design has numerically been proven to have the most effective flow mechanism in terms of preventing carbon deposition. Nevertheless, further design improvements should be considered to completely eliminate particles on the cavity walls.

Table 5 Temperature results and DPM results of the optimized seeded $1 \mathrm{~kW}$ cavity receiver designs (Rosin-Rammler parameters at the inlet: $d_{p, m}=7.167 \mu m ; n=0.759$ )

\section{SCR-0 SCR-I SCR-II SCR-III}

Temperature results

$\begin{array}{ccccc}\mathrm{T}_{\text {avg,3D }}[\mathrm{K}] & 665 & 709 & 692 & 706 \\ \mathrm{TNU}_{3 \mathrm{D}}[\%] & 10.0 & 10.5 & 11.5 & 10.4 \\ \mathrm{~T}_{\text {max,walls }}[\mathrm{K}] & 991 & 1100 & 1023 & 1027 \\ \mathrm{~T}_{\text {avg,outlet }}[\mathrm{K}] & 811 & 864 & 932 & 928\end{array}$

\section{DPM results}

$\begin{array}{lllll}\mathrm{c}_{\text {avg,walls }}\left[\mathrm{kg} / \mathrm{m}^{3}\right] & 0.0177 & 0.0169 & 0.0142 & 0.0172\end{array}$

\section{CONCLUSION}

In this paper, a CFD study is presented which investigates solid phase particles suspended in the main flow and their effect on wall deposition and heat transfer inside the fluid domain by using the discrete phase model. The validation of the DPM-enabled CFD model, which can be considered to be mainly qualitative, showed agreements in terms of deposition patterns on the window. Hence, similar DPM parameters were applied to the prior CFD model of the $1 \mathrm{~kW}$ solar cavity receiver. Outcome of the cold conditions parametric study depicted following conclusions:

- While heat transfer and temperature uniformity of the bulk flow were enhanced as a result of the particle-laden flow, deposition of the heavier particles were observed at the bottom of the wall. 
- Higher flow rates of the main feed would increase temperature uniformity and

- Particle loading proportionally affects the amount of deposition at the bottom of the receiver.

Including irradiation of the receiver lead to the following conclusions for the parametric design iteration study:

- Considering possible alterations of the parameters that are independent of the receiver geometry or flow configuration led to the use of a finer particle size distribution for the particle feed. Results showed a decrease of particle deposition and enhancement of temperature uniformity in the fluid domain.

- The thermal hot spot observed in the original SCR-0 receiver design became more significant in the SCR-I design because of the radiative beam directly irradiating the cavity wall. Elongation of the frustum in SCR-II allowed to spread the radiative flux, which positively affected the wall temperature as well as the convective heating of the flowing medium.

- Modification of the reactor geometry from a cylindrical body in SCR-0 to a combined cylinderconical body in SCR-I to SCR-III diminished the Rankine-vortex priorly present in the fluid domain. Nevertheless, both the reduction of carbon deposition and outlet gas temperature were favoured by the newly proposed designs.

The CFD study presented in this work pin-pointed areas of optimization for solar cavity receiver-reactors which adopt active particle seeding for heat transfer enhancement purposes. In particular, this study highlighted the positive impact of receiver elongation with respect to highly directional and concentrated irradiation of the cavity. Moreover, the presented results advocate the importance of using the finest particle sizes as possible while using maximizing the flow rate and maintaining an acceptable particle loading to minimize particle deposition. The outcome obtained from this study can serve as a guideline for the design process of particle-seeded solar reactors.

\section{Acknowledgement}

This research has been funded by Impulse Fund Project No. IMP/14/049 of KU Leuven and campus De Nayer of KU Leuven. Special thanks to Mostafa Abuseada of University of California Los Angeles for his valuable assistance and feedback on the present work. 


\section{References}

[1] S. Izquierdo, C. Montañs, C. Dopazo, and N. Fueyo, "Analysis of CSP plants for the definition of energy policies: The influence on electricity cost of solar multiples, capacity factors and energy storage," Energy Policy, vol. 38, no. 10, pp. 6215-6221, 2010.

[2] M. Roeb, M. Neises, N. Monnerie, C. Sattler, and R. Pitz-Paal, "Technologies and trends in solar power and fuels," Energy and Environmental Science, vol. 4, no. 7. pp. 2503-2511, 2011.

[3] A. M. Daabo, E. Bellos, S. Pavlovic, M. Anser, S. Mahmoud, and R. K. Al-dadah, "Characterization of a micro thermal cavity receiver - Experimental and analytical investigation," Therm. Sci. Eng. Prog., vol. 18, no. April, p. 100554, 2020.

[4] A. M. Daabo, A. Ahmad, S. Mahmoud, and R. K. Al-dadah, "Parametric analysis of small scale cavity receiver with optimum shape for solar powered closed Brayton cycle applications," Appl. Therm. Eng., vol. 122, pp. 626-641, 2017.

[5] A. M. Daabo, S. Mahmoud, and R. K. Al-dadah, "The optical efficiency of three different geometries of a small scale cavity receiver for concentrated solar applications," Appl. Energy, vol. 179, pp. 1081-1096, 2016.

[6] R. Loni, B. Ghobadian, A. B. Kasaeian, M. M. Akhlaghi, E. Bellos, and G. Najafi, "Sensitivity analysis of parabolic trough concentrator using rectangular cavity receiver," Appl. Therm. Eng., vol. 169, no. April 2019, 2020.

[7] S. Rodat, S. Abanades, J. L. Sans, and G. Flamant, "Hydrogen production from solar thermal dissociation of natural gas: development of a $10 \mathrm{~kW}$ solar chemical reactor prototype," Sol. Energy, vol. 83, no. 9, pp. 1599-1610, 2009.

[8] R. Guil-lopez, J. A. Botas, J. L. G. Fierro, and D. P. Serrano, "Applied Catalysis A : General Comparison of metal and carbon catalysts for hydrogen production by methane decomposition," "Applied Catal. A, Gen., vol. 396, no. 1-2, pp. 40-51, 2011.

[9] D. Trommer, D. Hirsch, and A. Steinfeld, "Kinetic investigation of the thermal decomposition of 
CH4by direct irradiation of a vortex-flow laden with carbon particles," Int. J. Hydrogen Energy, vol. 29, no. 6, pp. 627-633, 2004.

[10] N. Muradov, F. Smith, and A. T-Raissi, "Catalytic activity of carbons for methane decomposition reaction," in Catalysis Today, 2005, vol. 102-103, pp. 225-233.

[11] M. Brkic, E. Koepf, and A. Meier, "Solar carbothermal reduction of aerosolized ZnO particles under vacuum : Modeling, experimentation, and characterization of a drop- tube reactor," Chem. Eng. J., vol. 313, pp. 435-449, 2017.

[12] G. Maag, G. Zanganeh, and A. Steinfeld, "Solar thermal cracking of methane in a particle-flow reactor for the co-production of hydrogen and carbon," Int. J. Hydrogen Energy, vol. 34, no. 18, pp. 7676-7685, 2009.

[13] A. Chinnici, M. Arjomandi, Z. F. Tian, Z. Lu, and G. J. Nathan, “A Novel Solar Expanding-Vortex Particle Reactor : Influence of Vortex Structure on Particle Residence Times and Trajectories," Sol. Energy, vol. 122, pp. 58-75, 2015.

[14] J. L. Pinilla et al., "Metallic and carbonaceous -based catalysts performance in the solar catalytic decomposition of methane for hydrogen and carbon production," Int. J. Hydrogen Energy, vol. 37, no. 12, pp. 9645-9655, 2012.

[15] N. Özalp, "Hydrogen Production with Zero Emissions Footprint: Challenges and Solutions Towards Commercialization,” NATO Sci. Peace Secur. Ser. C Environ. Secur., 2013.

[16] M. Kogan and A. Kogan, "Production of hydrogen and carbon by solar thermal methane splitting. I. The unseeded reactor," Int. J. Hydrogen Energy, vol. 28, no. 11, pp. 1187-1198, 2003.

[17] A. Kogan, M. Kogan, and S. Barak, "Production of hydrogen and carbon by solar thermal methane splitting. II. Room temperature simulation tests of seeded solar reactor," Int. J. Hydrogen Energy, vol. 29, no. 12, pp. 1227-1236, 2004.

[18] A. Kogan and M. Kogan, "The tornado flow configuration-an effective method for screening of a solar reactor window,” J. Sol. Energy Eng. Trans. ASME, vol. 124, no. 3, pp. 206-214, 2002. 
[19] J. Rutten and J. Verschoren, "Design, manufacturing and experimental testing of a mechanical exit port mechanism for a solar reactor," KU Leuven, 2018.

[20] H. F. Lutro, "The effect of thermophoresis on the particle deposition on a cylinder," Institut for fysikk, 2012.

[21] A. Kogan, M. Israeli, and E. Alcobi, "Production of hydrogen and carbon by solar thermal methane splitting. IV. Preliminary simulation of a confined tornado flow configuration by computational fluid dynamics," Int. J. Hydrogen Energy, vol. 32, no. 18, pp. 4800-4810, 2007.

[22] D. Jaya Krishna and N. Ozalp, "Numerical investigation of particle deposition inside aero-shielded solar cyclone reactor: A promising solution for reactor clogging," Int. J. Heat Fluid Flow, vol. 40, no. October, pp. 198-209, 2013.

[23] B. Nezzari, R. Gomri, M. Constantine, and L. De G, "Study of cracking of methane for hydrogen production using concentrated solar energy," Int. J. Hydrogen Energy, vol. 45, no. 1, pp. 135-148, 2019.

[24] N. Ozalp and A. Kanjirakat, "A computational fluid dynamics study on the effect of carbon particle seeding for the improvement of solar reactor performance," J. Heat Transfer, vol. 132, no. 12, pp. $1-7,2010$.

[25] C. K. Ho, “A review of high-temperature particle receivers for concentrating solar power," Appl. Therm. Eng., vol. 109, pp. 958-969, 2016.

[26] D. Hirsch and A. Steinfeld, "Radiative transfer in a solar chemical reactor for the co-production of hydrogen and carbon by thermal decomposition of methane," Chem. Eng. Sci., vol. 59, no. 24, pp. 5771-5778, 2004.

[27] C. Ophoff, N. Ozalp, and D. Moens, "Monte Carlo ray tracing coupled CFD modelling and experimental testing of a $1 \mathrm{~kW}$ solar cavity receiver radiated via $7 \mathrm{~kW}$ HFSS," in ASME 2019 Heat Transfer Summer Conference, HT 2019, collocated with the ASME 2019 13th International Conference on Energy Sustainability, 2019, no. July. 
[28] ANSYS, "Ansys software inc.".

[29] S. Bellan, K. Matsubara, C. Hyun, N. Gokon, and T. Kodama, "CFD-DEM investigation of particles circulation pattern of two-tower fl uidized bed reactor for beam-down solar concentrating system," Powder Technol., vol. 319, pp. 228-237, 2017.

[30] D. W. Green and R. H. Perry, Perry's Chemical Engineers 'Handbook, 8th ed. McGraw-Hill, USA, 2008.

[31] ANSYS, “Fluent Theory Guide 17.0.” 2016.

[32] ANSYS, “Fluent 6.0 User’s Guide - Discrete PhaseModels,” 2001.

[33] V. Shilapuram, D. Jaya Krishna, and N. Ozalp, "Residence time distribution and flow field study of aero-shielded solar cyclone reactor for emission-free generation of hydrogen," Int. J. Hydrogen Energy, vol. 36, no. 21, pp. 13488-13500, 2011.

[34] D. Hirsch and A. Steinfeld, "Solar hydrogen production by thermal decomposition of natural gas using a vortex-flow reactor," Int. J. Hydrogen Energy, vol. 29, no. 1, pp. 47-55, 2004.

[35] P. Rosin and E. Rammler, "The Laws Governing the Fineness of Powdered Coal.-J. Institute of Fuel, 7: 29-36. Evaluation of the Folk and Ward graphic measures," J. Sediment. Res., vol. 48, pp. 863878, 1933.

[36] C. K. Ho, S. S. Khalsa, and N. P. Siegel, "Modeling on-sun tests of a prototype solid particle receiver for concentrating solar power processes and storage," in Proceedings of the ASME 3rd International Conference on Energy Sustainability 2009, ES2009, 2009, vol. 2, pp. 543-550.

[37] E. Koepf, "Design, modeling and demonstration of a novel receiver/reactor for the high temperature decomposition of $\mathrm{ZnO}$ using concentrated sunlight,” 2013.

[38] C. Ophoff, M. Abuseada, N. Ozalp, and D. Moens, "Systematic approach for design optimization of a 3 kW solar cavity receiver via multiphysics analysis," Sol. Energy, vol. 206, pp. 420-435, 2020.

[39] H. H. Klein, R. Rubin, and J. Karni, "Experimental evaluation of particle consumption in a particle 
seeded solar receiver," in Journal of Solar Energy Engineering, Transactions of the ASME, 2008, vol. 130, no. 1, pp. 0110121-0110128.

[40] I. Brezzani, “Rosin-Rammler Diagram Plotting Tool (Matlab).”.

[41] W. Peng et al., "Flow pattern in reverse-flow centrifugal separators," Powder Technol., vol. 127, no. 3, pp. 212-222, 2002. 